\title{
Robin Who? Zwischen materiellen Interessen, wertgestützten Ideen und nationalen Institutionen: Die Europäische Finanztransaktionssteuer in Deutschland und Großbritannien
}

\author{
Aukje van Loon iD
}

Angenommen: 5. Juli 2021 / Online publiziert: 16. August 2021

(C) Der/die Autor(en) 2021

Zusammenfassung Als Reaktion auf die Eurokrise brachte die Europäische Union (EU) mehrere Reformpakete und Vorschläge zur Überarbeitung des GovernanceRahmens der Wirtschafts- und Währungsunion (WWU) auf den Weg. Diese Studie untersucht den Vorschlag zur Stärkung der Finanzregulierung im Zusammenhang mit der Debatte über Finanztransaktionssteuern (FTS), die sogenannte Robin-HoodSteuer, und die divergierenden politischen Reaktionen der britischen und deutschen Regierungen. Besonderes Augenmerk wird auf die Auswirkungen der Pluralität der Akteure und die issue salience bei den Prozessen zur Bildung von Präferenzen in diesen Ländern gelegt. Hauptziel ist es festzustellen, wer die Reaktionen dieser Regierungen während der FTS-Debatte bestimmt hat und warum finanzielle und nicht-finanzielle gesellschaftliche Akteure bei der Entscheidungsfindung entweder beachtet oder abgelehnt wurden. Durch die Anwendung des Societal Approach als Theorie endogener Präferenzformation werden drei erklärende Variablen, materielle Interessen, wertgestützte Ideen und nationale Institutionen, die in der Innenpolitik der Länder dominieren, untersucht, um zu berücksichtigen, wann diese von Bedeutung sind, wie sie interagieren und welche bei der Gestaltung der FTS-Positionen dieser Regierungen prägend sind. Diese vergleichende theoriegeleitete empirische Analyse zeigt, dass Gewerkschaften, Wähler*innen und NGOs in Deutschland in der Lage waren, wichtige Interessen der Finanzindustrie bei der Gestaltung der Regierungsposition zur Unterstützung der vorgeschlagenen Reform zu umgehen, während diese Akteure in Großbritannien, einer einheitlichen Finanzindustrie, die sich vehement gegen die Steuer aussprach, nicht erfolgreich entgegenwirkten. Anknüpfend an die aktuelle Literatur zur europäischen Finanzregulierung nach der Krise, die sich mit einem gewissen Dissens zwischen einer Zunahme und einer Abnahme der

Dr. Aukje van Loon $(\square)$

Lehrstuhl für Internationale Politik, Fakultät für Sozialwissenschaft, Ruhr-Universität Bochum,

Bochum, Deutschland

E-Mail: aukje.vanloon@rub.de 
demokratischen Politikgestaltung in Krisenzeiten befasst, soll diese Untersuchung ein umfassendes Verständnis der Präferenzbildung der Regierungen fördern und eine gewisse verstärkte demokratische Politikgestaltung erkennen.

Schlüsselwörter Akteurspluralität · Präferenzbildung · Wirtschafts- und Währungsunion · Issue Salience · Societal Approach · Transaktionssteuer

\title{
Robin Who? Between material interests, value-based ideas and national institutions: The European financial transaction tax in Germany and Great-Britain
}

\begin{abstract}
Prompted by the Euro crisis, the European Union (EU) introduced multiple reform packages and proposals in order to overhaul the Economic and Monetary Union (EMU) governance framework. This study recalls the proposal of strengthening financial regulation surrounding the financial transaction tax (FTT) debate, or the so-called Robin Hood tax, and its most extreme governments' policy responses stemming from Germany and the UK. Particular attention is paid to the impact of actor plurality and issue salience in these countries' domestic preference formation processes. Principal aim is to ascertain who determined these governments' responses during the FTT debate and why financial and non-financial societal actors were either paid attention to or rejected in the process of decision making. By applying the societal approach to governmental preference formation, three explanatory variables, material interests, value-based ideas and domestic institutions, dominant in the countries' domestic politics, are investigated in order to account for when these mattered, how they interacted and which prevailed in shaping these governments' FTT positions. This comparative empirical analysis reveals that in Germany trade unions, voters and NGOs were able to circumvent narrow financial industry interests in shaping its government's position towards supporting the proposed reform, whereas in the UK these actors were not able to counter a unified financial industry overwhelmingly opposing the tax. Contributing to contemporary post-crisis European financial governance literature, this examination seeks to advance a comprehensive understanding of governmental preference formation, reading a certain dissent between an increase versus a decrease of democratic policy making.
\end{abstract}

Keywords Actor plurality · Domestic preference formation · Economic and Monetary Union · Issue salience · Societal approach · Transaction tax

\section{Einleitung und Puzzle}

Die Eurokrise hat gezeigt, dass der Governance-Rahmen der Wirtschafts- und Währungsunion (WWU) unvollständig ist, was dazu führte, dass die Europäische Kommission 2011 mehrere Reformpakete und Vorschläge zur Stärkung der Finanzregulierung vorlegte. Dies führte zu Konflikten zwischen den EU-Mitgliedstaaten über den Inhalt und die Gestaltung dieser Reformen. Ursprünglich war Finanzregulierung, eine eher technokratische Tätigkeit, nur für die Akteure attraktiv, die an der 
Steuerung des WWU-Rahmens beteiligt waren. Das unmittelbare Rampenlicht auf ihre Unvollständigkeit wurde durch eine erhöhte issue salience, d.h. inwieweit die Bedeutung eines Themas von Akteuren geteilt wird, gekennzeichnet und löste öffentliche Diskussionen und eine breite Akteurspluralität (Interessengruppen, Gewerkschaften, NGOs und Wähler*innen) aus. Die Debatte, welche Akteure bei den Reaktionen auf Regulierungsreformen während der Verhandlungen auf EU-Ebene eine Rolle spielten, wurde dadurch wiederbelebt (Kastner 2018, S. 1651). Hutter und Kriesi (2019, S. 997) weisen darauf hin, dass ,conflicts over Europe have been transferred from the backrooms of political decision-making to the public sphere". In der aktuellen europäischen Integrationsliteratur werden diese Aspekte der issue salience und der Pluralität der Akteure in der Regel durch die Verwendung theoretischer Perspektiven wie liberalen Intergouvernementalismus (Schimmelfennig 2015), Neofunktionalismus (Niemann und Ioannou 2015) oder des postfunktionalistischen Ansatzes (Puetter 2012) in Einklang gebracht. Während in den meisten Studien die Positionen europäischer Institutionen oder Mitgliedstaaten bei der Erklärung der Politikgestaltung in der Eurokrise im Mittelpunkt stehen (Degner und Leuffen 2019a; Fabbrini und Puetter 2016), nimmt die Forschungslücke zu den Positionen der europäischen Mitgliedsregierungen beim Rekonstruieren der WWU allmählich ab (Degner und Leuffen 2019b; Rothacher 2015). Vergleichsstudien sind jedoch noch relativ spärlich (Schirm 2018; Van Loon 2020).

Diese Studie leistet einen Beitrag zur Literatur der Europäischen Integration und der Europäischen Politischen Ökonomie in Bezug auf die Finanzregulierung nach der Eurokrise und versucht, ein umfassendes Verständnis der Präferenzbildung von Regierungen zu fördern. Diese Literatur befasst sich mit einem gewissen Dissens zwischen einer Zunahme und einer Abnahme der demokratischen Politikgestaltung. Dieser Artikel baut auf dieser Literatur auf und geht davon aus, dass das europäische Krisenmanagement nicht dazu geführt hat, dass Regierungen mit einem gewissen Moment der Autonomie in der Politikgestaltung auf nationaler Ebene konfrontiert wurden (Csehi und Puetter 2020). Stattdessen waren die Regierungen dadurch, dass sie nicht in einem innenpolitischen Vakuum handelten, gegenüber endogenen gesellschaftlichen Akteuren responsiv, was zu einer verstärkten demokratischen Politikgestaltung führte. Van Loon (2018) untersucht die EU-Finanzaufsicht und- Regulierung und argumentiert, dass die nationale Ebene für die Entscheidungsfindung der EUPolitik von entscheidender Bedeutung ist. Die Untersuchung der gesellschaftlichen Einflüsse auf die Reformpositionen der Regierungen ist daher eine wichtige Voraussetzung, um zu verstehen, wie und warum bestimmte divergierende nationale Positionen auf der EU-Ebene verfolgt werden. Ein zentraler Aspekt betrifft den Prozess der Bildung innerstaatlicher Präferenzen, der mit einem zumindest wahrgenommenen Verlust demokratischer Entscheidungsfindung in Krisenzeiten verbunden ist (Csehi und Puetter 2020). Doch auf wen haben die Regierungen angesichts der Pluralität der Akteure und der issue salience während der FTS-Reformdiskussionen reagiert und warum?

Diese Studie untersucht den Vorschlag zur Stärkung der europäischen Finanzregulierung, insbesondere im Zusammenhang mit der (2011-2014) Finanztransaktionssteuerdebatte (FTS), der sogenannten Robin Hood-Steuer, und beleuchtet die divergierende Responsivität, d.h. die politischen Reaktionen der britischen und deutschen 
Regierungen, um eine verstärkte demokratische Politikgestaltung zu analysieren. ${ }^{1}$ Ausgelöst durch die globale Finanzkrise Ende 2007, die sich bei Ausbruch der Eurokrise 2010 ausbreitete, schlug die Europäische Kommission 2011 eine europäische FTS vor, welche bis 2014 umgesetzt werden sollte, um sicherzustellen, dass der Finanzsektor seinen gerechten Anteil und erhebliche Beiträge zur Deckung der Krisenkosten und zur wirtschaftlichen Erholung leistet. In der Erwartung, dass die Steuerzahler den Finanzsektor in Krisenzeiten unterstützen und gleichzeitig dem Finanzsektor ermöglichen würden, die Vorteile des Missmanagements zu nutzen, wurde das Thema von den Mitgliedsregierungen umfassend debattiert. Entweder sie sprachen sich nachdrücklich für die Steuer aus, vor allem Deutschland, oder sie lehnten ihre Einführung vehement ab, zum Beispiel das Vereinigte Königreich. Aufgrund der Varianz in ihren FTS-Positionen wurden diese Mitgliedsregierungen ausgewählt, um in einer vergleichenden empirischen Analyse ihre Responsivität gegenüber verschiedenen gesellschaftlichen Akteuren zu analysieren.

Im Jahr 2013 einigten sich elf Mitgliedsregierungen auf die Stärkung der Finanzregulierung im Rahmen des Verfahrens des sogenannten erweiternden Kooperationsmechnismus (,„enhanced cooperation procedure“). Nach einem Jahrzehnt erfolgloser Verhandlungen setzen vor allem Deutschland und Frankreich die FTS regelmäßig auf die Agenda des ECOFIN (EU-Finanzministerrat), um das Thema voranzubringen und das politische Engagement der restlichen Mitgliedsregierungen zu erneuern. Aktuelle Entwicklungen scheinen diesen Prozess zu beschleunigen, da der Brexit und der Ausschluss des Vereinigten Königreichs als „foot-dragger“ (Quaglia 2017, S. 1) bei der Blockierung der FTS-Verhandlungen sowie die aktuelle COVID19-Pandemiekrise die deutsche Ratspräsidentschaft 2020 dazu veranlasst haben, die EUMitglieder zu „Solidarität, Kooperation und gemeinsamen Lösungen“ aufzurufen, um den EU-Haushalt zu finanzieren (Bundesministerium der Finanzen 2020). Die endgültige Vereinbarung steht jedoch zum Zeitpunkt des Schreibens noch aus.

Diese Studie wird sich insbesondere mit den Auswirkungen von issue salience und Akteurspluralität befassen, die zur Einbeziehung eines breiten Spektrums von endogenen gesellschaftlichen Akteuren, einschließlich Interessengruppen, Gewerkschaften, NGOs und Wählern*innen, führten und die den britischen und deutschen Prozess der endogenen Präferenzformation prägten. Das Hauptziel besteht darin herauszufinden, wer die Reaktionen dieser Regierungen während der FTS-Debatte bestimmte und warum finanzielle und nicht-finanzielle gesellschaftliche Akteure bei der Gestaltung von Regierungspositionen entweder beachtet oder ignoriert wurden. Durch Anwendung des Societal Approach als Theorie endogener Präferenzformation (Schirm 2011, 2018, 2020) werden drei unabhängige Variablen, materielle Interessen, wertgestützte Ideen und nationale Institutionen, die in der Innenpolitik der Länder dominieren, untersucht, um zu berücksichtigen, wann und wie sie interagieren und unter welchen Bedingungen sie die Regierungspositionen als abhängige Variable geprägt haben. Diese vergleichende Analyse zeigt, dass die deutsche Regierung die Anliegen von Gewerkschaften, NGOs und Wähler*innen, welche wichtige Interessen der Finanzindustrie umgehen konnten, in ihre FTS-Position einbezog, während

\footnotetext{
${ }^{1}$ Dieser Artikel ist eine Weiterentwicklung der bisherigen Forschung zur Anwendung des Societal Approach auf die FTS-Fallstudie (van Loon 2020, 2021).
} 
die genannten Akteure in Großbritannien nicht in der Lage waren, einer einheitlichen Finanzindustrie, die sich überwiegend gegen die FTS aussprach, entgegenzuwirken.

Der nächste Abschnitt bietet einen Überblick über die Literatur zur europäischen Finanzregulierung nach der Eurokrise. Diese verdeutlicht einen gewissen Dissens zwischen einer Zunahme und einer Abnahme der demokratischen Politikgestaltung und der Responsivität der Regierungen während der EU-Reformverhandlungen im Allgemeinen und der FTS im Besonderen. Es folgt ein kurzer Überblick über die zentralen Theorien endogener Präferenzbildung, gefolgt von der Einführung und Weiterentwicklung des Societal Approach, einer Erläuterung der innovativen Elemente, einer Definition der Variablen und einer Formulierung einiger Kernhypothesen. Daraufhin wird die FTS-Reform kurz vorgestellt, gefolgt von einer eingehenden Fallstudie zur endogenen Präferenzformation, in der die unabhängigen Variablen, materielle Interessen, wertgestützte Ideen und nationale Institutionen, analysiert werden. Der Artikel endet mit einer Zusammenfassung der empirischen Ergebnisse.

\section{Europäische Literatur zur Finanzregulierung und demokratischer Politikgestaltung}

In der aktuellen Literatur zur europäischen Finanzregulierung nach der Eurokrise werden Regulierungsreforminitiativen erörtert, welche in der Regel nicht der demokratischen Legitimität entsprechen. Die Ansicht, dass Lösungen für das Krisenmanagement eher Kriterien für einen Output waren, bei dem die EU-Institutionen in den Entscheidungsprozess eingebunden waren, als für die Legitimität des Inputs, d.h. die Einbeziehung gesellschaftlicher Akteure in diesen Prozess, stand im Vordergrund (Kreuder-Sonnen 2016). Dieses Ungleichgewicht ist auf die Eurokrise zurückzuführen, die eine Situation von erheblicher Dringlichkeit, Unsicherheit und unbekannten Folgen geschaffen hat, in der schnell und entschlossen gehandelt werden musste, woraus ein anschließendes Demokratiedefizit zu resultieren schien. Kreuder-Sonnen (2018, S. 962) erklärt:

crises typically do imply a broader than usual menu of feasible policy options (...) as a critical threat to a referent community opens the way for policy-makers to employ extraordinary means to cope with the situation, because the sense of crisis induces public deference to claims of political necessity.

Das Umfeld und die Dynamik der europäischen Finanzregulierung in Bezug auf die Beziehungen zwischen der nationalen und der supranationalen Ebene sowie deren Folgen für Legitimität in der EU werden in der neueren Literatur wiederholt. Es wird argumentiert, dass die europäische Finanzregulierung nach der Eurokrise einen bedeutenden, supranationalen Charakter bei der Stärkung der EU-Institutionen erhalten hat, d.h. die Europäische Kommission wurde angesehen als ,,an unexpected winner of the crisis" bei der Umsetzung und Anwendung der Governance-Regeln (Bauer und Becker 2014). Auch die verstärkte europäische Führungsrolle der Zentralbank während der Eurokrise verdeutlicht diese Perspektive (Verdun 2017). Umgekehrt argumentiert die neue Intergouvernementalismus-Literatur, dass das Krisenmanagement der Kommission dazu veranlasste, teilweise von der Gemeinschaftsmethode 
abzuweichen und stattdessen ,de novo bodies“ zu stärken (Bickerton et al. 2015, S. 705). Obwohl Niemann und Ioannou (2015) die zunehmende Bedeutung der zwischenstaatlichen Koordinierung im Rahmen des Europäischen Rates während und nach der Eurokrise hervorheben, argumentieren Smeets und Beach (2019, S. 2), dass ihr ,informal and isolated character“ die Kontrolle der Mitgliedsregierungen marginalisiert und „created more instead of less dependence on EU institutions“. Schmidt (2015) argumentiert, dass das Versäumnis dieser Institutionen, während der Eurokrise Ergebnisse zu erzielen, ohne den Input der Öffentlichkeit in den Reformen einzubeziehen, die demokratische Legitimität verschlechterte und damit das langjährige Demokratiedefizitproblem der EU widerspiegelt. Andere Literatur zeigt jedoch die positiven Auswirkungen der Eurokrise auf explizite Aspekte der Demokratie auf nationaler Ebene. Laut Kriesi (2018) führte die Unzufriedenheit der Bürger*innen mit der wirtschaftlichen Leistung ihres Landes während der Eurokrise zu einer Stärkung der demokratischen Grundsätze. Cordero und Simón (2016) stellen gleichermaßen fest, dass die Unzufriedenheit mit der nationalen Wirtschaft dennoch zu Rettungsaktionen führte (z.B. die Euro-Rettungspakete für Griechenland), die einen gewissen Einfluss auf die Unterstützung der Bürger*innen für demokratische Werte in den betroffenen Ländern hatten. Während einige Untersuchungen Krisenreaktionen veranschaulichen, welche sich negativ auf die demokratische Legitimität der europäischen Finanzregulierung ausgewirkt haben, betrachtet ein anderer akademischer Diskurs krisenbedingte Maßnahmen auf nationaler Ebene, die auf eine Verbesserung der demokratischen Prinzipien hinweisen.

Dieser Artikel stimmt mit dem letztgenannten Literaturstrang überein und stellt die Behauptung in Frage, dass ,,when failure hits as in the Euro crisis, [...] all sources of legitimacy suddenly, simultaneously collapse“ (Weiler 2012, S. 837). Das Argument von Degner und Leuffen (2019b), dass die Eurokrise die Legitimität der Position der Regierungen, insbesondere in der ersten Phase der europäischen Integration, der Bildung innerstaatlicher Präferenzen, tatsächlich verbessert haben könnte, wird unterstützt. Die dringende und unsichere Krisensituation führte zu einem besonderen Umfeld politischer Auseinandersetzungen, einer sogenannten Politisierung (De Wilde et al. 2016). Dies spiegelt einen Prozess wider, der ,from permissive consensus towards constraining dissensus“ führt und gleichzeitig ,,beyond interest group bargaining into the public sphere" gelangt (Hooghe und Marks 2009, S. 5). Angesichts der Tatsache, dass sich die Responsivität der Regierungen darauf bezieht, dass gewählte Politiker verschiedenen Akteuren mit weitreichenden Themen Priorität einräumen, ist es insbesondere in Krisenzeiten interessant zu untersuchen, welche Forderungen der gesellschaftlichen Akteure in die Position der Regierung einbezogen und welche Bedenken während des Prozesses der demokratischen Politikgestaltung zurückgewiesen wurden. Ein zentrales Element endogener Präferenzbildung ist die Symbiose und Interaktion zwischen Gruppen der Finanzindustrie und der Öffentlichkeit, welche darauf abzielen, die Regierung für sich einzunehmen, um eine Stärkung der Finanzregulierung entweder zu akzeptieren oder abzulehnen.

Die traditionelle Literatur der Internationalen (Europäischen) Politischen Ökonomie reflektiert die Auswirkungen der Interessengruppenpolitik auf die Bildung innerstaatlicher Präferenzen (Grossman und Helpman 1994). Darüber hinaus berichtet die Literatur zu Interessengruppen (Dür und Mateo 2016; Eising 2009) sowie 
der liberale Intergouvernementalismus (Moravcsik 1993), dass die gut organisierten und ausgestatteten Interessengruppen einen privilegierten institutionellen Zugang zu Regierungen genießen, die als ,transmission belt“ bei der Verteidigung ihrer Präferenzen auf der Suche nach einem Abkommen auf EU-Ebene dienen. Mit etablierten Informationskanälen und einer Expertise in Bezug auf relevante Themen sind die Regierungen tendenziell auf nationale Interessengruppen ausgerichtet. Studien zur FTS von Kalaitzake (2017) sowie von Hardiman und Metinosy (2019) betonen, dass bei der Ausübung struktureller Macht, d.h. der wirtschaftlichen Interdependenz des Finanzsektors von einer nationalen Wirtschaft (Exporte, Arbeitsplätze und Bruttoinlandsprodukt (BIP)) und instrumenteller Macht, d.h. institutionelle Vernetzung im Sinne einer aktiven Lobbyarbeit der Regierung, Gruppen der Finanzindustrie ihre Präferenzen sicherten, indem sie den Reformvorschlag in den meisten EU-Mitgliedstaaten verzögerten und abschwächten. Ezrow und Hellwig (2014) argumentieren daher, dass Regierungen eher auf Marktakteure als auf Wähler*innen reagieren. Dies wird von Sandres und Toka (2013, S. 22) unterstützt, die Folgendes feststellen:

[i]n sum, in determining their own stances towards the EU, political elites appear to place more weight on the views of the economically rich and powerful [interest groups] than they do on the views of their own constituents.

Das Management der Eurokrise, das issue salience und unbekannte Konsequenzen mit sich brachte, führte jedoch dazu, dass die Bürger*innen hoch besorgt und gut über die Positionen ihrer jeweiligen Regierungen in den EU-Reformverhandlungen informiert waren. Laut Hobolt und Klemmensen (2008, S. 310) wird von den Regierungen in Situationen hoher politischer Auseinandersetzungen erwartet, dass sie einen größeren Impuls haben, der öffentlichen Meinung zu folgen. Culpepper (2012) stellt fest, dass der Wechsel von ,quiet to noisy politics“ zu einer stärkeren Responsivität der Regierungen auf die Forderungen der Bürger*innen führt, was gleichzeitig zu einer Verringerung der Fähigkeit der Interessengruppen führt, die Positionen der Regierungen zu formen. Die strukturelle Macht der Finanzinteressen führt jedoch nicht automatisch zu instrumenteller Macht. Insbesondere in Zeiten von ,noisy politics“ sind Regierungen weniger responsiv für Präferenzen der Finanzbranche und eher auf wahlorientierte Prioritäten eingestellt (Keller 2018). Hooghe und Marks (2009, S. 18) argumentieren gleichermaßen, dass „,mass politics trump interest group politics when both come into play". Medienanalysen von Kastner (2017) und Degner und Leuffen (2019b) haben gezeigt, dass die issue salience der Finanzregulierung in der Öffentlichkeit insbesondere in den Jahren 2011 und 2013 zugenommen hat. Dies spiegelt sich weitgehend in den britischen Steuerkampagnen „Robin Hood Tax“ und der deutschen „Steuer gegen Armut“ wider, bei denen eine breite Mitgliederzahl von NGOs einschließlich Entwicklungs-, Umwelt- und Religionsorganisationen sowie Gewerkschaften erreicht wurde. Ihr Ziel war es, breite Unterstützung für ihre Ziele zur Einführung der Steuer durch Lobbyarbeit bei ihren jeweiligen Regierungen zu sammeln.

Die Literatur zur europäischen Finanzregulierung nach der Krise ist letztlich geteilt, ob das Euro-Krisenmanagement und die Entscheidungen der EU-Institutionen und der Mitgliedstaaten die demokratische Politikgestaltung reduziert oder gestärkt haben. Ebenso enthält sie unterschiedliche Erklärungen zur Bildung der 
Regierungspositionen. Die Literatur ist sich zudem uneins, ob die Regierungen aufgrund der Krisensituationen einen erheblichen Entscheidungsspielraum hatten oder ob sie während der Bildung innerstaatlicher Präferenzen auf endogene gesellschaftliche Akteure reagierten. Diese Studie folgt letzterem Argument. Die Eurokrise betraf unmittelbar beide Arten von Akteuren, finanzielle und nicht-finanzielle, die entweder für oder gegen eine Stärkung der Finanzregulierung waren, was wiederum zu politischen Auseinandersetzungen über die Einführung von Steuern auf Finanztransaktionen führte. In Krisenzeiten sahen sich die Regierungen mit einer erheblichen Notwendigkeit konfrontiert, in sehr begrenzter Zeit auf wesentliche Reformen $\mathrm{zu}$ drängen. Um jedoch eine Bestrafung an der Wahlurne zu vermeiden, waren sie ebenso verpflichtet, die Befindlichkeiten der gesellschaftlichen Akteure zu berücksichtigen. Daher wurde der Entscheidungsspielraum der Regierungen ,in determining EMU stances (...) clearly constrained by domestic politics“ (Van Loon 2020, S. 220). Die Frage, welche finanziellen und nicht-finanziellen gesellschaftlichen Akteure letztendlich die divergierenden FTS-Positionen der britischen und deutschen Regierung während dieses Prozesses der demokratischen Politikgestaltung geprägt haben, ist daher von großer Relevanz.

\section{Analytischer Rahmen: Interessen, Ideen und Institutionen im Societal Approach}

Diese Studie verwendet den Societal Approach als Theorie endogener Präferenzformation (Schirm 2011, 2018, 2020), da sie sich auf die gesellschaftliche Erklärung von Regierungspolitik, materielle Interessen, wertgestützte Ideen und nationale Institutionen konzentriert, die die Regierungspositionen vor internationalen oder zwischenstaatlichen Verhandlungen dominieren (Schirm 2013, S. 690). Der Societal Approach ermöglicht somit nicht nur das Öffnen, sondern auch eine explizite Entfaltung der Black Box, um die Variation der britischen und deutschen FTS-Positionen zu erklären (Van Loon 2020). Bei der Anwendung und Erweiterung der Theorien endogener Präferenzformation wie des IB-Liberalismus (Moravcsik 1997) oder Domestic-Politics-Ansätze wie die Konzeptionalisierung innenpolitischer Faktoren zur Erklärung der Außenwirtschaftspolitik (Goldstein und Keohane 1993; Keohane und Milner 1996), den Historischen Institutionalismus (Fioretos 2011) sowie verschiedener „Varieties of Capitalism“ (Hall und Soskice 2001), wird in dieser Studie als Analysewerkzeug der Societal Approach eingesetzt. Er wurde ,developed as a complementary approach“ (Schirm 2020, S. 400), und stellt eine innovative Weiterentwicklung und Verfeinerung (refinement) dieser Theorien dar. Ähnlich wie bei diesen Theorien wird davon ausgegangen, dass Regierungen in demokratischen politischen Systemen beabsichtigen, im Amt zu bleiben und daher ,democratic governments tend to be responsive and accountable to voters, this is, to societal preferences" (Schirm 2013, S. 690). Daher spiegeln ihre Positionen die Erwägungen der endogenen gesellschaftlichen Akteure wider. Im Gegensatz zur Betrachtung der Bedeutung von entweder Interessen, Ideen oder Institutionen bezieht dieses Analyseinstrument jedoch alle drei unabhängigen Variablen ein, um Regierungspositionen als abhängige Variable zu erklären (Schirm 2016, S. 68, 2020, S. 404). 
In ,linking social preferences and state behavior“ liefert Moravcsik (1997, S. 515) eher statistische Varianten des Liberalismus, den kommerziellen, den ideellen und den republikanischen. Es werden weder das Zusammenspiel gesellschaftlicher Erwägungen noch die Bedingungen berücksichtigt, unter denen Regierungen entweder materielle oder ideelle Befindlichkeiten in ihre innerstaatliche Präferenzbildung einbeziehen. Die Rolle, die nationale Institutionen bei der Privilegierung einiger gesellschaftlicher Anliegen gegenüber anderen spielen, und wie diese Unterschiede formen, sind von ebenso geringer Bedeutung. Eine systematische Untersuchung der individuellen Rolle materieller Interessen, wertgestützter Ideen und nationaler Institutionen bei der Unterstützung oder Gegenüberstellung sowie ihrer Interdependenz ist ein entscheidender innovativer Aspekt des Societal Approach. Folglich erfordert die Bestimmung, welche dieser erklärenden Variablen überwiegend für Unterschiede zwischen den Positionen der Regierungen verantwortlich sind, einen weiteren Schritt, um erklären zu können, warum in einigen Fällen materielle Interessen bei der Gestaltung der Positionen der Regierung dominieren, während wertgestützte Ideen und nationale Institutionen in anderen Situationen prägend sind. Schirm (2020, S. 397) stellt fest, dass diese Konzeption in früheren innenpolitischen Ansätzen bisher nicht enthalten war und, dass letztere „left room for a systematic exploration of the conditions under which each of the three individual domestic forces matters more than the other(s) in shaping governmental preferences".

In Anlehnung an die Forschungsergebnisse früherer Wissenschaftler*innen schreibt der Societal Approach bestimmte Bedeutungen den gesellschaftlichen Akteuren und Strukturen zu. Aufbauend auf Milner (1997) und Moravcsik wird die Variable materielle Interessen als kurzfristige Verteilungsberechnungen der Wirtschaftssektoren definiert, die sich rasch an Veränderungen in der internationalen Wirtschaft anpassen. Wertgestützte Ideen sind definiert als langfristige, gemeinsame Erwartungen der Wähler*innen an ein angemessenes Regierungsverhalten bei der Steuerung der Wirtschaft (z.B. Vertrauen in die Marktkräfte im Vergleich zu staatlicher Regulierung). Im Vergleich zu den materiellen Interessen folgen diese Ideen einer bestimmten Pfadabhängigkeit und können sich unter veränderten (wirtschaftlichen) Bedingungen nicht schnell anpassen. Die Definition der nationalen Institutionen erweitert die Betrachtung langfristiger institutioneller Rahmenbedingungen durch Fioretos (2011) als Grundlage für den Wettbewerbsvorteil der Wirtschaftsgruppen. Dies gilt auch für Hall und Soskice (2001), wenn es darum geht, diese als formale Regelungen für die sozioökonomische Koordinierung zu identifizieren und dabei unterschiedliche Vorschriften der Marktwirtschaft (z.B. koordinierte Marktwirtschaft (KME) gegenüber liberaler Marktwirtschaft (LME)) zu beleuchten. Nationale institutionelle Komplementaritäten konkurrieren nicht mit materiellen Interessen oder wertgestützen Ideen. Tatsächlich prägen sie die Anliegen der gesellschaftlichen Akteure und damit die Positionen der Regierungen: Von Institutionen als Regulierungsrahmen wird erwartet, dass sie die Grundüberzeugungen der Gesellschaft in Bezug auf das Verhältnis zwischen Staat und Markt widerspiegeln, sowie Zielen der Regulierung der Finanzmärkte und wirtschaftlichen Überlegungen für einen Regulierungsrahmen, der dem Wettbewerbsvorteil einer Volkswirtschaft entsprechen. 
Die explizite Spezifikation der Variablen unterstützt die Artikulation einzelner Hypothesen. Schirm (2009, S. 9; 2020, S. 404) weist darauf hin, dass diese nicht veranschaulichen, ob eine Variable anstelle einer anderen wichtig ist, sondern sich auf die Bedingungen konzentrieren, unter denen jede einzelne Variable bei der Gestaltung der Regierungsposition entscheidender wird. Diese zentralen Hypothesen werden wie folgt konzipiert und im Rahmen der Stärkung der Finanzregulierung eingefügt. Die erste Hypothese erwartet, dass sich Interessengruppen und Gewerkschaften aus den Bereichen Finanzen und Industrie bei der Gestaltung der FTS-Position der Regierung durchsetzen: Wenn sich die Stärkung der EU-Finanzregulierung direkt auf bestimmte Wirtschaftssektoren auswirkt und potenzielle Kosten-NutzenBerechnungen impliziert, ist es wahrscheinlicher, dass materielle Interessen die Position der Regierung beeinflussen, weil Lobbyarbeit die Präferenzbildung prägt. Die zweite Hypothese beschreibt die Rolle von Wähler*innen und NGOs bei der Präferenzbildung der Regierung: Wenn die Stärkung der EU-Finanzregulierung grundlegend-bedeutende ("fundamental-salient", Schirm 2020, S. 404) und langfristige gesellschaftliche Erwartungen an die angemessene Rolle einer Regierung bei der Steuerung der Wirtschaft mit sich bringt, setzen sich eher wertgestützte Ideen durch bei der Gestaltung der Position der Regierung. Diese beiden Hypothesen zeigen, dass die erklärenden Variablen konkurrieren können. Eine dritte Hypothese befasst sich mit ihrer Interdependenz: Wenn die Stärkung der EU-Finanzregulierung sowohl potenzielle Kosten-Nutzen-Berechnungen für bestimmte Wirtschaftssektoren als auch grundlegende und langfristige gesellschaftliche Erwartungen an die angemessene Rolle der Regierung bei der Steuerung der Wirtschaft betrifft, konkurrieren oder verstärken sich die Variablen gegenseitig bei der Gestaltung der FTS-Position der Regierung. Zudem besagt die vierte Hypothese, dass wenn die Stärkung der Finanzregulierung Fragen zu formalen Regelungen der sozioökonomischen Koordinierung betrifft, werden die Positionen der Regierungen außerdem mit den nationalen Institutionen übereinstimmen. Die Auswirkung materieller Interessen und wertgestützter Ideen auf die Präferenzbildung der Regierung wird verstärkt, wenn sie nationalen „institutional settings“ entsprechen (Schirm 2016, S. 69).

Im Sinne theoretischer Fokussierung (parsimony) konzentriert sich der Societal Approach auf Interessen, Ideen und Institutionen als Primärvariablen und ,conceptually deepens a crucial but limited part of the political process" (Schirm 2020, S. 409). Andere potenziell wichtige Faktoren, wie z. B. politische Parteien und Parteiprogramme, werden nicht explizit untersucht, da die gesellschaftlichen Interessen und Ideen, die die Parteiprogramme prägen, über Meinungsumfragen oder Aussagen von Interessengruppen berücksichtigt werden. Weiterhin gehört zur Konzeption des Societal Approach auch die Pluralität sowohl materiell als auch ideell motivierter Akteure und infolgedessen die Möglichkeit von „,de facto alignments“ von spezifischen materiellen Interessen mit spezifischen ideellen Erwartungshaltungen die ähnliche Forderungen an die Regierung haben und mit entgegengesetzten Forderungen anderer gesellschaftlicher Interessen und Ideen konkurrieren können (Schirm 2020, S. 407-409).

Diese Studie befasst sich mit den Entwicklungen nach der Eurokrise im spezifischen Kontext der Finanzregulierung: Da der Nachhall der Krise die Regierungspolitik zunehmend beeinflusst hat, löste dies sowohl eine issue salience als 
auch die Pluralität der Akteure (Kastner 2018) mit nicht-finanziellen Akteuren aus, die durch eine Reihe von ideell motivierten Zielen mobilisiert wurden und sehr daran interessiert waren, die FTS-Positionen ihrer jeweiligen Regierungen zu gestalten. Um ein breiteres Spektrum nationaler Interessengruppen berücksichtigen zu können, die unmittelbar von der Einführung einer Steuer auf Finanztransaktionen betroffen sind, und die Reaktion der jeweiligen Regierungen auf ihre Präferenzen, werden weitere nationale Akteure in die Analyse aufgenommen (Van Loon 2018). Die materiell motivierten Branchenverbände werden durch Gewerkschaften ergänzt, die als Quelle für Interessen gelten, und ideell motivierte Wähler*innen werden von NGOs als Quelle für Ideen ergänzt. Gewerkschaften sind auf Mitgliedschaft basierende Interessengruppen, die in der Regel einen hervorragenden Zugang zu Entscheidungsträger*innen haben. Dies liegt hauptsächlich daran, dass sie politisch nützliche Ressourcen erhalten (Dür und Mateo 2012, S. 972), die gegen Zugang und günstige politische Entscheidungen ausgetauscht werden können. Diese Akteure können aufgrund ihrer sektoralen Relevanz und des Beitrags des gesamten Bruttoinlandsprodukts (BIP) zur Wirtschaft eines Landes eine privilegierte Position im Entscheidungsprozess einnehmen. Im Gegensatz zu sektoralen Interessenverbänden und Gewerkschaften sind die Anreize von NGOs, sich am Prozess der Bildung Regierungspräferenzen d.h. Steuer-, Sozial-, Umwelt-, und Verteilungsfragen zu beteiligen, eher ideell motiviert. Angesichts diffuser Kosten und Nutzen konzentrieren sich sogenannte Outside-Lobbying-Aktivitäten auf die Gestaltung der öffentlichen Meinung durch Demonstrationen, Informationsveranstaltungen, Pressemitteilungen, Internetkampagnen und Petitionen (Dür und Mateo 2016, S. 1). Die eingeschränkte Fähigkeit, die Position der Regierungen durch sogenanntes Inside-lobbying zu gestalten (De Bruycker und Beyers 2019), ist nicht nur den im Vergleich zu Interessengruppen weniger direktem Kontakt und Zugang zu Entscheidungsträger*innen zurückzuführen, sondern auch darauf, dass NGOs normalerweise nicht über für die Gestaltung von Strategien notwendigen Ressourcen verfügen, mit denen sie Investitionen oder Beschäftigungen zurückziehen können. Schiffers und Körner (2019) argumentieren jedoch, dass weder Lobbying-Aktivitäten für Interessengruppen noch für NGOs bestimmt sind, sondern dass jeder Akteurstyp eine Strategie anwenden oder beide kombinieren kann, um die Positionen der Regierungen zu formen. Politische Auseinandersetzungen und issue salience können, wie die Forschung zur Medienberichterstattung zeigt (Epstein und Segal 2000), zu einer Stärkung der Finanzregulierung führen. Dies schafft ein größeres öffentliches Bewusstsein, das Entscheidungsträger*innen dazu motivieren kann, aus Sorge vor einem Legitimitätsverlust im Zusammenhang mit dem Ziel, im Amt zu bleiben, zu reagieren.

In der empirischen Analyse wird eine qualitative vergleichende Fallstudie verwendet, die sich auf eine Diskursanalyse stützt, welche relevante Interessen, Ideen und Institutionen bezogene Indikatoren für die Präferenzbildung der Regierungen während der FTS-Debatte 2011-2013 verfolgt. Dies umfasst den Zeitraum der vorgeschlagenen Finanztransaktionsrichtlinie und die anschließende Einführung des erweiternden Kooperationsmechanismus (,enhanced cooperation procedure“) Die Relevanz der drei erklärenden Variablen für die unterschiedlichen Positionen der Regierungen wird untersucht, indem ermittelt wird, welche gesellschaftlichen Akteure, Branchenverbände, Gewerkschaften, Wähler*innen und NGOs, unmittelbar 
von dem FTS-Vorschlag betroffen waren, wie sie in den Präferenzbildungsprozessen interagierten und sich bei der Gestaltung der FTS-Positionen der Regierungen durchgesetzt haben. Im Mittelpunkt stehen die Aussagen dieser Akteure in Pressemitteilungen, Positionspapieren, Meinungsumfragen, offiziellen Websites und Sekundärquellen mit dem Ziel, die inhaltlichen Ursprünge und Bedenken im Kern der vorgeschlagenen Steuerreform zu ermitteln.

\section{Die deutschen und britischen Regierungspositionen zur Finanztransaktionssteuer}

Nachdem der G20-Gipfel in Toronto 2010 keine Einigung über global koordinierte Maßnahmen zur Betreuung des Finanzsektors erzielt hatte, schlug der Kommissionspräsident, José Barroso, im September 2011 eine Richtlinie vor, um eine harmonisierte, breit angelegte FTS als Reaktion auf die globalen Finanzmärkte zu schaffen ,as a question of fairness (...) for the financial sector to make a contribution back to society“ (Chaffin et al. 2011). Als Beispiel für eine mögliche globale Umsetzung sollte die FTS von den Mitgliedstaaten übernommen werden. Die Hauptziele dabei waren: (1) Vermeidung einer Fragmentierung des Binnenmarktes für Finanzdienstleistungen; (2) sicherzustellen, dass Finanzinstitute einen fairen und wesentlichen Beitrag zur Deckung der Kosten der Krise leisten; (3) angemessene negative Anreize für Transaktionen einzuführen, die die Effizienz der Finanzmärkte nicht verbessern; und (4) ergänzende Regulierungsmaßnahmen zur Vermeidung künftiger Krisen zu schaffen (Europäische Kommission 2011, S. 2). Viele Mitgliedsregierungen bestritten die FTS hauptsächlich wegen des Risikos, das Wachstum und die Verlagerung des Finanzsektors zu behindern. Als sich herausstellte, dass es problematisch war, die erforderliche Einstimmigkeit für die Verabschiedung des Vorschlags zu erreichen, wurden die widerstrebendsten Regierungen wie Schweden, die Niederlande und Großbritannien, vor allem von Deutschland und Frankreich umgangen, als sie die Kommission aufforderten, den erweiterten Kooperationsmechanismus (,,enhanced cooperation procedure") einzuführen (van Loon 2020).

\subsection{Die Transaktionssteuer aus deutscher Sicht - mit oder ohne Großbritannien}

Die Bundesregierung hat eine breit angelegte FTS unterstützt, und als 2010 ein Konsens darüber erzielt wurde, war dies der Hauptgrund für die Aufforderung an die Kommission, den Mechanismus der verstärkten Zusammenarbeit umzusetzen (Bundesregierung 2014). Im November 2011 wies Bundesfinanzminister Wolfgang Schäuble gegnerische Argumente zurück:

The objections made by some who claim it would mean a substantial drop in employment and in the economy generally seem to rest on exaggerated (...) projections and, more important, ignore the potential of such a tax to stabilize currency markets in a way to boost rather than damage the real economy (Winnet et al. 2011). 
Durch die Zusammenfassung der Steuerziele führte Schäuble Folgendes auf: (1) Der Finanzsektor muss zur Deckung der Krisenkosten beitragen, (2) es würden erhebliche Steuereinnahmen erzielt und (3) Maßnahmen der Finanzmärkte begrenzt. Die Begründung der FTS lautete ,not only a question of the economy and the budget but of democratic legitimacy“ und möglicherweise 2 Mrd. $€$ pro Jahr für Deutschland. Schäuble erklärte: „I'd prefer to have it in my budget but it's better to have it for climate change or development aid than to have nothing" (Elliot und Kollewe 2011).

In Ermangelung einer einstimmigen Einigung beschleunigte die Bundesregierung den Prozess, indem sie die Einführung von Finanztransaktionssteuern durch verstärkte Zusammenarbeit beantragte. Auf einem ECOFIN-Treffen kündigte Schäuble dies an (FAZ 2012) und würde sich auch ohne diesen Mechanismus ,bemühen, im Rahmen der zwischenstaatlichen Zusammenarbeit in möglichst vielen Mitgliedstaaten Steuern zu erzielen“ (Bundesregierung 2012, S. 2). Bundeskanzlerin Angela Merkel erklärte das starke Engagement der Regierung, insbesondere nachdem sie von der SPD und den Grünen unterstützt wurde (Handelsblatt 2012):

We support the introduction of a financial transaction tax, because the people in our countries still have the impression (...) that the financial sector must make an appropriate contribution to managing the costs of the financial crisis, and the financial transaction tax will be levied precisely for this purpose (Bundesregierung 2014).

Die FTS wurde 2013 in den Koalitionsvertrag aufgenommen. Unter besonderer Betonung von ,,[n]o financial market, no financial product, no financial market player without supervision“. Der Schwerpunkt lag auf einer breit angelegten FTS im Rahmen der verstärkten Zusammenarbeit in der EU. Dies sollte rasch umgesetzt werden, um die Beteiligung des Finanzsektors an den Kosten der Krise zu stärken, indem alle Finanzinstrumente einbezogen wurden: Aktien, Anleihen, Investmentaktien, Devisentransaktionen und Derivatekontrakte. Bei der Gestaltung der Steuer betont die Regierung, wie wichtig es ist, die Auswirkungen der Steuer auf ,die Altersvorsorge, Kleinanleger und die Realwirtschaft (...) zu bewerten und gleichzeitig unerwünschte Formen der Finanztransaktionen zu reduzieren“ (Koalitionsvertrag 2013, S. 46).

\subsection{Die Finanztransaktionssteuer aus britischer Sicht - lebendig und tödlich}

Die britische Regierung hat 2011 eine permanente Bankabgabe eingeführt, um den Banken die implizite Rettungsgarantie in Rechnung zu stellen. Sie lehnte den FTSVorschlag entschieden ab und bestand auf ihre globale Umsetzung (Chaffin et al. 2011) und argumentierte, dass sie aufgrund der Anwendung der Steuer, die auf der Grundlage des Aufenthaltsprinzips definiert wird, verschiedene negative Auswir- 
kungen auf die Volkswirtschaft der EU und Großbritanniens haben würde. ${ }^{2}$ Dies würde dazu führen, dass der Finanzdienstleistungssektor seine Geschäftstätigkeit in Regionen verlagert, in denen keine Steuern erhoben werden, was die Wettbewerbsfähigkeit der Branche verringert, das Wachstum in der EU und in Großbritannien beeinträchtigt und zum Verlust von Arbeitsplätzen führt (Hoban 2012). Der britische Premierminister David Cameron zitierte die Folgenabschätzung der Kommission von 2011, indem er darauf hinwies, dass eine europäische FTS das BIP der EU um 200 Mrd. $€$ senken und fast 500.000 Arbeitsplätze kosten und einen Großteil der Finanzindustrie aus Europa verdrängen könnte: „Even to be considering this at a time when we are struggling to get our economies growing is quite simply madness“ (Cameron 2012). Die britische Regierung äußerte ferner Bedenken hinsichtlich der negativen Auswirkungen einer FTS auf die britische Finanzindustrie. In einer Rede auf der Global Investment Conference argumentierte Cameron, ,,we shouldn't spend our time in politics endlessly bashing banks and financial institutions" und hielt die FTS für inakzeptabel:

We want an economy to recover, if you want an economy to grow you have to play to your strengths. And I think this leads to two quite important points that we need to make clear to our European colleagues. Unless it is introduced in the same way all over the world, this is a mistake for Europe. It is a mistake for Europe (...) not just because we are a financial centre here in Britain, but we actually account for $40 \%$ of Europe's financial services. This is an industry that serves not just Britain, but serves Europe (Cameron 2013).

Der Schatzkanzler Georg Osborne untermauert dieses Argument mit der Feststellung, dass Vorschläge für eine FTS ,are a bullet aimed at the heart of London, (...) economic suicide for Britain and for Europe [and] this government will ensure that our national interests and our voice in the EU are protected" (Falloon 2011). Auf der ECOFIN-Sitzung im Januar 2013 enthielt sich das Vereinigte Königreich der Stimme, um die FTS zu billigen als die Annahme mit qualifizierter Mehrheit genehmigt wurde (Baker 2013). Auf verschiedene Anfragen der Mitgliedsregierungen hin gab die Kommission einen Monat später einen zweiten FTS-Vorschlag heraus. Dieser war dem ursprünglichen sehr ähnlich, durch die Stärkung der AntiUmsiedlungs-Aspekte und durch die Einbeziehung des Emissionsprinzips, eines der Hauptanliegen Großbritanniens, stieß die britische Regierung erneut auf Missbilligung über die Auswirkungen der Steuer auf nicht teilnehmende Mitgliedstaaten. Die Anfechtung der FTS-Legalität aufgrund exterritorialer Aspekte wurde der Vorschlag im April 2014 vom Europäischen Gerichtshof abgelehnt (Parker 2014). Aufgrund dieser Entwicklung wurde die FTS später als ,alive and deadly“ bezeichnet (House of Lords 2013).

\footnotetext{
2 Die wesentliche Voraussetzung für die Besteuerung einer Transaktion im Rahmen des ersten FTS-Vorschlags der Kommission ist, dass mindestens eine Partei der Transaktion in einem EU-Mitgliedstaat ansässig ist und dass sein Finanzinstitut, das an der Transaktion beteiligt oder Vermittler bei der Transaktion ist im Hoheitsgebiet eines EU-Mitgliedstaats niedergelassen ist. In ihrem zweiten FTS-Vorschlag im Jahr 2013 fügte die Kommission ein Emissionsprinzip hinzu.
} 


\section{Issue Salience und Akteurspluralität}

Finanz- und Unternehmensgruppen in Deutschland und Großbritannien lehnten den FTS -Vorschlag vehement ab. Tab. 1 zeigt den Unterschied in der Bedeutung des Finanzsektors für Deutschland und das Vereinigte Königreich in Bezug auf den Beitrag des Finanzsektors zu Arbeitsplätzen, Exporten und Bruttowertschöpfung in einer ausgewählten Anzahl von Volkswirtschaften der europäischen Mitgliedstaaten. Auf den ersten Blick könnte dies erklären, warum die britische Regierung mit einem großen Finanzsektor und ihr deutsches Pendant mit einem kleineren Finanzsektor gegensätzliche Positionen hatten. Eine solche Erklärung wäre jedoch zu einfach. Sowohl deutsche als auch britische Finanz- und Wirtschaftsinteressenverbände sprachen sich deutlich gegen die FTS aus und besaßen strukturelle und instrumentelle Macht. Warum konnte die britische Finanzindustrie die Position ihrer Regierung gestalten, während die deutsche Regierung nicht so erfolgreich zu überzeugen war? Während die FTS einerseits regulatorische Auswirkungen auf die Finanztransaktionen hat, generiert sie andererseits erhebliche Einnahmen für Entwicklung und Umwelt. Dieser doppelte Zweck erklärt das Problem der Bedeutung und der Pluralität der Akteure im Zusammenhang mit den FTS-Debatten in Deutschland und Großbritannien.

\subsection{Deutschland: Materielle Interessen - Interessenverbände für Finanzen und Industrie}

In einem gemeinsamen Positionspapier äußerten acht deutsche Verbände ihre Zweifel, ob die FTS ihre Ziele erreichen könnte. Unter der Annahme, dass sich diese negativ auf den Finanzsektor und die Wirtschaft insgesamt auswirken würde, wurde allgemein befürchtet, dass dies (1) negative Auswirkungen auf Unternehmen und Arbeitnehmer haben und (2) die Wirtschaft belasten würde, insbesondere im Hinblick auf die Kreditversorgung, und (3) Finanzgeschäfte in schlecht regulierte Märkte verwandeln - mit Konsequenzen für die Marktstabilität - wenn sie nicht global oder zumindest EU-weit eingeführt würden (DIHK et al. 2011, S. 2). In der Zeit des Vorschlags der Kommission für die FTS im Rahmen einer verstärkten Zusammenarbeit teilten die deutschen Interessenverbände, der BDI, der BDB und die DIHK

Tab. 1 Finanzsektor in den Volkswirtschaften im Vergleich: Beschäftigung, Exporte und BWS, 2011

\begin{tabular}{lllll}
\hline & $\begin{array}{l}\text { Beschäftigung } \\
\text { (\% der } \\
\text { Gesamtbeschäftigung) }\end{array}$ & $\begin{array}{l}\text { Exporte } \\
\text { (\% des } \\
\text { Gesamtexports) }\end{array}$ & $\begin{array}{l}\text { BWS } \\
\text { (\% des Ge- } \\
\text { samt BWS })\end{array}$ & $\begin{array}{l}\text { Unterstützung } \\
\text { für FTS }\end{array}$ \\
\hline Niederlande & 2,6 & 6,6 & 8,5 & Nein \\
Großbritannien & 3,2 & 34,0 & 8,0 & Nein \\
Belgien & 1,0 & 8,1 & 5,6 & $\mathrm{Ja}$ \\
Frankreich & 1,1 & 7,1 & 4,3 & $\mathrm{Ja}$ \\
Österreich & 3,4 & 7,2 & 4,7 & $\mathrm{Ja}$ \\
Deutschland & 1,3 & 13,6 & 4,8 & $\mathrm{Ja}$ \\
\hline
\end{tabular}

Quelle: OECD (2020a); OECD (2020b); World Bank (2020) 
ihre Besorgnis über die negativen Auswirkungen auf die deutsche Realwirtschaft und die privaten Akteure. Die mögliche Abwanderung von Finanzinstituten, Banken und Investmentfonds in Länder, in denen keine Transaktionen besteuert werden, war ein Hauptanliegen, gefolgt von der Problematik der Kreditaufnahme deutscher Unternehmen und der Erwartung eines stagnierenden Wachstums und einer stagnierenden Beschäftigung. Die Ziele der FTS, den Finanzsektor an den Kosten der wirtschaftlichen Erholung zu beteiligen, aber auch die Vorsichtsmaßnahme für potenzielle künftige Kosten krisengeplagter Entwicklungen im Finanzsektor und die Schaffung von Anreizen für den spekulativen Handel, wurden kritisch bewertet. In einer öffentlichen Anhörung des Finanzausschusses des Deutschen Bundestages 2011, lehnte der deutsche Bankenverband diese Ziele ab und befürchtete negative Auswirkungen auf die Wirtschaft mit der Feststellung, dass ,,selbst die EU-Kommission einen Verlust des Bruttoinlandsprodukts von $1,76 \%$ bei einer EU-weiten Steuer von $0,1 \%$ auf Aktien (0,01\% auf Derivate), erwartet" (Deutscher Bundestag 2011). Die FTS würde nicht nur Finanzinstitute betreffen, sondern alle Käufer*innen von Finanzprodukten, einschließlich kleiner Sparer. Der BDI, der deutsche Industrieverband, war außerdem der Ansicht, dass die FTS ,diejenigen, die die Kosten der geschätzten Steuereinnahmen der EU von $57 \mathrm{Mrd} . €(.$.$) tragen, die Bürger und die$ Realwirtschaft sein werden“ (Deutscher Bundestag 2011). In einer vom deutschen Aktieninstitut in Auftrag gegebenen Studie wurde die Belastung privater Haushalte und Unternehmen auf 5,0 bis 7,3 Mrd. € pro Jahr geschätzt (DAI 2013, S. 5). Der Investmentfondsverband BVI gab an, dass ,der Kleininvestor in Deutschland, der nicht ins Ausland ziehen kann, der Dumme ist" (Bankenverband 2013).

Im Gegensatz dazu begrüßte der DGB, der Gewerkschaftsbund, die Richtlinie und plädierte für eine breit angelegte FTS. Da die globale Finanz- und Eurokrise zu einer steigenden Arbeitslosigkeit geführt hatte, war er sich der sozialen und politischen Konsequenzen bewusst, da die Bürger*innen das Vertrauen in die Märkte verloren hatten (DGB 2011a, S. 3). Seine Position war, dass ,financial market players that were chiefly responsible for the biggest financial and economic crisis of the past 80 years [yet] solely the taxpayers and workers (...) have borne the chief burden of overcoming the crisis“ (DGB 2013, S. 9). Die fehlgeleiteten Strategien der Banken und die zunehmende Ungleichheit hatten Finanzspekulationen ausgelöst, die zu dem Hauptproblem der mangelnden Kontrolle über die Marktakteure geführt hatten, was die Notwendigkeit einer stärkeren Intervention der Regierung implizierte. Im Februar 2011 forderte er politische Stabilität, wirtschaftlichen Wohlstand und soziale Sicherheit:

[f]inancial markets must not only be monitored, but also effectively regulated.

(...) Those who cause the crisis must be asked to pay. That is why we need a financial transaction tax (...). Furthermore, all financial market products must be audited for their economic benefit (...). The same regulatory rules must apply for all financial market players (DGB 2011a, S. 7).

Claus Matecki, Vorstandsmitglied des DGB, erklärte die Dringlichkeit der Umsetzung der FTS auf nationaler Ebene: ,if the desired introduction of the tax on an international or European level does not seem feasible in the short term, Germany (...) must send a clear signal to the other EU member states“ (DGB 2011b). Der 
DGB schlug einen „Marshall-Plan für wirtschaftliche Impulse, Investitionen und Entwicklung“" und die FTS vor, um Einnahmen zu erzielen, die den Mitarbeitern, der Umwelt, den Ländern und der Realwirtschaft zugutekommen (DGB 2013, S. 10).

\subsection{Großbritannien: Materielle Interessen - Interessenverbände für Finanzen und Industrie}

In einem gemeinsamen Brief an George Osborne forderten elf britische Finanzunternehmen die britische Regierung auf, eine starke Position gegen die Steuer einzunehmen und sich eher auf „Projekte zur Steigerung von Beschäftigung und Wirtschaftswachstum“ zu konzentrieren, da sie die festgelegten Ziele nicht erreichen würde und eine verheerende Auswirkung nicht nur, ,within the financial system (...) but for the vast array of end users“ (ISDA 2011) hätte. In einem offenen Brief äußerten die Gruppen der Finanzbranche ihre deutliche Opposition und kritisierten, dass die FTSZahlen der Kommission ,fehlerhaft“ seien und, dass die Steuer ,the largest financial derivatives market in the world, with an average daily turnover in interest rate derivatives of just over \$1.4 trillion“" (ISDA 2012). Ohne eine weltweite Einführung hätte die FTS-Steuer schädliche Auswirkungen auf Großbritannien und insbesondere auf London als Europas größtes Finanzzentrum und die europäischen Finanzmärkte im Allgemeinen, wodurch die EU gegenüber anderen Handelszentren im Wettbewerb benachteiligt würde und damit die Finanzaktivitäten zu anderen Gerichtsbarkeiten umgeleitet würden (CBI 2012, S. 1; COBCOE 2011, S. 2). Chris Cummings, Chief Executive von TheCityUK, verwies auf die Stärke und Größe der City of London als europäisches Finanzzentrum und bezeichnete die Finanztransaktionssteuer als eine nicht wettbewerbsfähige Maßnahme, die nachteilige Auswirkungen habe. Dies ,,is the exact opposite of the type of intervention that is needed at the moment if the European economy is to be stimulated and if we are to show international markets that Europe is open for business“" (City AM 2014). Neil Bentley, der stellvertretende Generaldirektor des CBI, wiederholte die Bedenken hinsichtlich der finanziellen Umleitung und bezeichnete die FTS als ein ,crude instrument“:

To consider the introduction of a financial transaction tax at a time when we should be totally focused on promoting growth is a mistake. Such a tax could have the opposite effect, increasing the cost of capital for businesses and holding back their growth potential (Wearden 2011).

In einer vom EU-Unterausschuss für Wirtschaft und Finanzen und internationalen Handel des britischen Parlaments durchgeführten Aufforderung zur Einreichung von Beweismitteln zur FTS wurden die Gruppen der Wirtschafts- und Finanzbranche in ihre Positionen vereinheitlicht (House of Lords 2012). Besonderes Augenmerk wurde auf die vermuteten negativen Auswirkungen einer FTS auf die Realwirtschaft gelegt, wobei betont wurde, dass dies

would actually weaken both financial sector operators and the economies in which they do business, which would ultimately be detrimental to European tax revenue, to employment and to the citizens of the EU (BBA 2011, S. 5). 
Dementsprechend applaudierten die untersuchten britischen Verbände für Finanzen und Unternehmensinteressen der britischen Regierung offen, nachdem diese die Ablehnung einer FTS klargestellt hatte und nannten sie ,,absolutely the right decision not to adopt the European Financial Transaction Tax in the UK" (CBI 2012, S. 1). Da Pensionsfonds verschiedene Produkte verwenden, die unter die Finanztransaktionssteuer fallen, argumentierten Finanz- und Industriegruppen, dass die FTS den Steuerzahlern tatsächlich mehr schaden würde als dem Finanzsektor. Die AIMA argumentierte, dass sie ,would affect EU taxpayers most, by reducing savings and retirement income“ (House of Lords 2012, S. 8). Die Gewerkschaft Unite, forderte die Regierung auf, die FTS zu unterstützen, indem sie erklärte, dass die Finanzindustrie weiterhin Gewinne erzielte, während die finanziellen Kosten der Steuerzahler für die Rettungsaktion $955 \mathrm{Mrd} . £$ betrugen. ,[T]he finance sector can give something back to society“ (House of Lords 2012, S. 207). Owen Tudor, Leiter der Abteilung für Europäische Union und Internationale Beziehungen der Gewerkschaft TUC sagte:

we believe it would generate much needed revenue for global public goods, in particular tackling climate change and helping to meet the costs of any deal agreed under the UNFCCC process, helping to achieve the millennium development goals and combating poverty and the need for damaging public service cuts in advanced economies (House of Lords 2012, S. 104).

\subsection{Unterstützende deutsche versus geteilte britische öffentliche Meinung}

Die Medienanalyse als Indikator für eine Steigerung des Stellenwerts im Fall der FTS, die beide untersuchten Länder vergleicht, zeigt, dass die Aufmerksamkeit der Medien in Deutschland im Zeitraum 2011-2013, also von dem Vorschlag der Kommission bis zur Entscheidung, die FTS im Rahmen einer verstärkten Zusammenarbeit umzusetzen, deutlich höher war als in Großbritannien. Weitere Belege für issue salience während der Präferenzbildungsprozesse zeigen, dass die deutschen in dieser Zeit zumindest stärker über dieses Problem informiert waren als die britischen Bürger: Auf die Frage „Have you ever heard of this tax on financial transactions“ antworteten $67 \%$ der deutschen gegenüber $33 \%$ der britischen Befragten mit ,yes“, während $33 \%$ gegenüber $63 \%$ mit „no“ antworteten (ITUC 2012).

Tab. 2 Wenn Sie über eine Reform der globalen Finanzmärkte nachdenken, sagen Sie mir bitte, ob Sie für oder gegen die folgenden Maßnahmen der EU sind. Die Einführung einer Steuer auf Finanztransaktionen $(\%)^{\mathrm{a}}$

\begin{tabular}{lllllllllll}
\hline 2011 & & \multicolumn{3}{c}{2012} & & \multicolumn{2}{c}{2013} \\
& $\begin{array}{l}\text { In } \\
\text { favour }\end{array}$ & Opposed & D.K. & & $\begin{array}{l}\text { In } \\
\text { favour }\end{array}$ & Opposed & D.K. & $\begin{array}{l}\text { In } \\
\text { favour }\end{array}$ & Opposed & D.K. \\
\hline EU & 61 & 25 & 14 & EU & 63 & 27 & 10 & 62 & 27 & 11 \\
DE & 74 & 16 & 10 & DE & 81 & 14 & 5 & 80 & 13 & 7 \\
$\mathrm{UK}$ & 43 & 41 & 16 & UK & 39 & 48 & 13 & 43 & 40 & 17 \\
\hline
\end{tabular}

Quelle: Eurobarometer (2011, S. 10; 2012, S. 144; 2013, S. 35)

a,Totally in favour“, ,totally opposed“ und ,don't know“ 
Die in Tab. 2 dargestellte tatsächliche FTS-Unterstützung spiegelt sich in den öffentlichen Umfragedaten wider, die im Zeitraum 2011-2013 aus dem Eurobarometer entnommen wurden. In Bezug auf die Fragen, ob die Befragten eine FTS befürworteten oder ablehnten, zeigten die deutschen Befragten eine höhere Zustimmungsrate für die europäische FTS, während britische Befragte in diesem Zeitraum über die Einführung der Steuer ziemlich gespalten waren. 2012 stieg die Zustimmungsrate der deutschen Befragten um 7\%, was dazu führte, dass Deutschland mit $81 \%$ die höchste Zustimmungsrate aller Länder (zusammen mit Österreich) hatte, wobei der Durchschnitt der EU 27 bei $63 \%$ lag. Die Missbilligungsrate in Großbritannien stieg ebenfalls um $7 \%$, wobei fast jeder Fünfte (48\%) gegen die FTS war. Die britische Öffentlichkeit war daher in diesem Thema eher gespalten, während die deutschen Bürger*innen eindeutig für die Einführung der Steuer waren.

Ein Grund für dieses Ungleichgewicht könnte darin liegen, dass die Befragten nach „den größten Bedrohungen für das wirtschaftliche Wohlergehen ihres Staates“ gefragt wurden. $87 \%$ der britischen Befragten, sahen in dem Mangel an Arbeitsplätzen eine große Bedrohung, während er im Vergleich dazu bei $70 \%$ der deutschen Befragten ganz oben auf der Liste stand. Die Macht der Banken wurde von $78 \%$ der deutschen Befragten und $65 \%$ der britischen Befragten als ernsthafte Bedrohung eingestuft (Pew Research 2012, S. 21). In Bezug auf die Frage nach der, ,angemessenen Rolle des Staates gegenüber dem Markt“ befürworteten 66,7\% der deutschen Befragten und 42,5\% der britischen Befragten die Aussage, dass ,governments should take more responsibility“, während eine Mehrheit von 56,6\% der britischen Befragten zustimmte, dass ,,people should take more responsibility“, verglichen mit nur $31 \%$ der deutschen Befragten (WVS 2006). Es muss jedoch angemerkt werden, dass die Zahlen von Pew Research auf eine größere Konvergenz zu einem ähnlichen Thema hindeuten: Eine Mehrheit der deutschen (62\%) und britischen Befragten $(55 \%)$ fand es wichtiger, dass der Staat garantiert, dass ,niemand in Not ist, als die Freiheit, die Ziele des Lebens ohne staatliche Einmischung zu verfolgen“ (Pew Research 2012, S. 1). Dies bezieht sich auf unterschiedliche Einstellungen zur individuellen Verantwortung im Gegensatz zur kollektiven Verantwortung: Während 63,6\% der deutschen Befragten der Meinung sind, dass die Einkommen einheitlich gemacht werden sollten, und nur 31,3\% der Ansicht sind, dass große Einkommensunterschiede als Anreize erforderlich sind, sind die britischen Befragten in diesem Bereich praktisch gespalten (49\% gegenüber 48,8\%) (WVS 2006). Die in diesen Zahlen ausgedrückten Unterschiede entsprechen den Marktwirtschaften der beiden Länder. Auch die Tatsache, dass Regierungen ,die Reichen besteuern und die Armen subventionieren“, wird von der überwiegenden Mehrheit der deutschen Befragten $(71,1 \%)$ und in geringerem Maße von den britischen Befragten $(59,9 \%)$ als wesentlicher Bestandteil der Demokratie angesehen (WVS 2006).

\subsection{Die ,Steuer gegen Armut““ Kampagne}

Unter dem Motto „Steuer gegen Armut“ konzentrierte sich diese von deutschen NGOs initiierte Kampagne hauptsächlich auf das Argument der Steuergerechtigkeit und die Gewinnung potenzieller FTS-Einnahmen. Sie könnten (1) dazu dienen, die Kosten für die Bekämpfung der Finanzkrise in erster Linie von der Finanzindustrie 
und nicht vom Steuerzahler zu belasten, und (2) die fortschreitende Wirkung der Steuer, die sich vor allem auf Einkommensstarke auswirkt, würde möglicherweise die zunehmenden Ungleichheiten bei der Einkommensverteilung verringern und somit als Entwicklungshilfe bei der Bekämpfung der nationalen und internationalen Armut dienen. Zweiunddreißig Unterzeichner entfachten diese Kampagne an, indem sie einen offenen Brief an die deutsche Regierung richteten. Dieser betonte die FTSVorteile: (1) sie verlangsamt und reguliert Finanztransaktionen an den Finanzmärkten, (2) sie umfasst alle spekulationsrelevanten Finanztransaktionen, (3) kurzfristige Transaktionen werden weniger rentabel gemacht, während mittel- und langfristige Investitionen einem niedrigen Steuersatz zwischen 0,1 und 0,01\% gegenüberstehen, und (4) Einnahmen werden für die Umsetzung der MDGs und für Entwicklungsmaßnahmen, die Bekämpfung von Armut sowie Klima- und Umweltschutz verwendet (Alt 2011). Der Kampagne gelang es, breite öffentliche Unterstützung zu erhalten, und sie erreichte im ersten Halbjahr 2011 eine beachtliche Mitgliederzahl. Gewerkschaften (einschließlich des DGB) setzten sich gemeinsam mit religiösen, Entwicklungs- und Umweltorganisationen für eine Verstärkung der Finanzregulierung ein. Als die Aussichten für eine globale und EU-weite Transaktionssteuer schwanden, begann eine starke Unterstützung der FTS für den erweiterten Kooperationsmechanismus (Wahl 2014, S. 6). Kampagnenforderungen wurden in Form einer Petition veröffentlicht, die innerhalb von vier Wochen von 66.000 Unterstützern unterzeichnet wurde (Alt 2011). Der deutsche Wahlkampfleiter Peter Wahl erklärte: „for civil society the process is nevertheless a great success“ da die Kampagne „Steuer gegen Armut" informelle und formelle ständige Kontakte zu Entscheidungsträgern hergestellt und die öffentliche Meinung mobilisiert hat, indem (1) öffentliche Veranstaltungen organisiert wurden, zu denen Entscheidungsträger eingeladen wurden, (2) formelle Petitionen organisiert und Unterschriften gesammelt wurden, (3) Briefe geschrieben und dadurch (4) Transparenz und Rechenschaftspflicht bei LobbyingAktivitäten unter Verwendung traditioneller Printmedien und des Internets geschaffen wurden (Wahl 2014, S. 14-15).

\subsection{Die ,Robin Hood Tax“ Kampagne}

Als die Einführung einer globalen Transaktionssteuer nachließ, mobilisierten britische NGOs gleichermaßen, um eine EU-weite Steuer zu unterstützen. Dies führte dazu, dass die britische Kampagne die „Robin Hood Tax“, ähnlich wie die deutsche Kampagne „Steuer gegen Armut“, Lobbyarbeit bei ihrer nationalen Regierung in Bezug auf die Frage einen erweiternden Kooperationsmechanismus (,enhanced cooperation procedure“) leistete. Einzelkampagnen wurden zu einer globalen Bewegung mit FTS-Unterstützung von einer Vielzahl von Organisationen sowie von Führungskräften aus Wirtschaft, Politik und Religion (CEPR 2013). Die britische Kampagne wurde im Februar 2010 von einer breiten Allianz von NGOs, Gewerkschaften, Ökonomen und Prominent*innen ins Leben gerufen und nahm Fahrt auf, als José Barroso im Herbst 2011 die EU-weite Finanztransaktionssteuer forderte (Stewart 2011). Sie erzeugte eine große (soziale) Medienberichterstattung und mobilisierte die öffentliche Unterstützung für die Finanztransaktionssteuer, mit der 20Mrd.£ zur Bekämpfung von Armut und Klimawandel im In- und Ausland aufge- 
bracht werden könnten, ohne den Finanzsektor zu beeinträchtigen (Wearden 2011). Im Jahr 2011 gab die Kampagne in ihrem ersten Policy Brief mit dem Titel ,There is an Alternative - Time for a Robin Hood Tax“ an, dass der effektivste Weg, um Geld als dem Finanzsektor zu sammeln, darin bestand, eine FTS von rund 0,05\% für alle Finanztransaktionen einzuführen, d.h. Aktien, Anleihen, Währungen und Derivate sowie $20 \mathrm{Mrd} . £$ sollten wie folgt ausgegeben werden; $10 \mathrm{Mrd}$. für den Schutz der Ärmsten in Großbritannien, 5 Mrd.£ für die Unterstützung der armen Länder und die Erreichung der Millenniums-Entwicklungsziehe (MDV) sowie 5 Mrd.£ für die Bekämpfung des Klimawandels (Robin Hood Tax 2011a, S. 5). David Hillman, Sprecher der „Robin Hood Tax“ Kampagne sagte:

Cameron and Osborne should be going to Europe with all guns blazing to protect the interests of the UK public, not to lobby on behalf of the City. An unrestrained financial sector is no basis for a secure economic future. The last financial crisis cost us at least $£ 1.8$ trillion according to the Bank of England the entire size of our GDP. It's time the banks paid their fair share (Robin Hood Tax 2011b).

Um sicherzustellen, dass die FTS-Einnahmen für Entwicklung und Klimawandel verwendet werden, forderte Mark Lawson, Leiter Entwicklungsfinanzierung bei Oxfam, eine Erhöhung des Drucks auf die deutsche Regierung sowie andere FTSUnterstützer, die ihre Ziele teilten (Oxfam 2011): „Given the power of Germany and France in the eurozone, a transaction tax at this level is now likely“ (Oxfam 2011, S. 12). Zu Wahls (2014, S. 13) Erfolg trug die sogenannte „Counter Expertise“ bei, welche seine Argumente legitimierte, da eine Reihe von Ökonomen Kampagnenvorschläge mit Studien und Berichten untermauerte - beispielsweise Untersuchungen von Schulmeister et al. (2008) -, die er auf seiner Website öffentlich zugänglich gemacht hat. Die Herausgabe unterschriebener Briefe und die Übergabe von Petitionen an Politiker*innen waren Aktivitäten, die die Bereitstellung schriftlicher Beweise während der Konsultation des Unterausschusses für Wirtschaft und Finanzen der EU und des internationalen Handels ergänzten.

\subsection{Koordinierte Marktwirtschaft (KMW) versus liberale Marktwirtschaft (LMW)}

Nationale Institutionen werden durch die Berücksichtigung langfristiger Komplementaritäten abgegrenzt, die sich aus zwei unterschiedlichen institutionellen Rahmenbedingungen ergeben, die von der Struktur der Volkswirtschaften geprägt sind: der LMW-KMW-Dichotomie sowie den unterschiedlichen Auffassungen der Beziehungen zwischen Regierung und Gesellschaft in Form von mehrheitlich wettbewerbsorientierter versus konsensbasierter Entscheidungsfindung (Hall und Soskice 2001, S. 33).

Das deutsche System der Finanzregulierung ist seit vielen Jahrzehnten auf die große Relevanz des verarbeitenden Gewerbes ausgerichtet. Dies äußerte sich in einem engen Branchen-Kapital-Zusammenhang mit einem System der sogenannten „Hausbanken“, das verbundenen Unternehmen langfristige Finanzmittel zur Verfügung stellte und es Unternehmen ermöglichte, langfristige Strategien zu verfolgen 
(Zimmermann 2010, S. 125). Dieses System wurde von einem korporatistischen Regulierungsrahmen begleitet, an dem Bund und Länder sowie die führenden deutschen Banken und Börsen beteiligt waren. Der Ansatz der Finanzregulierung konzentrierte sich daher auf die Bedürfnisse des verarbeitenden Gewerbes. Laut Zimmermann (2010, S. 132) kann die Förderung einer verstärkten und koordinierten Regulierung der Finanzmärkte durch die Bundesregierung als Strategie interpretiert werden, die darauf abzielt, neue Finanzakteure auf dem Radar zu halten, die Autonomie und Legitimität des Staates zu wahren sowie zu begrenzen Anpassungen im deutschen KMU-Sektor. Länder mit einem großen Finanzsektor profitieren von kurzfristig orientierten, volatilen Finanzmärkten, da sie mehr für eine erhöhte Anzahl von Finanztransaktionen verlangen und komplexere Finanzprodukte anbieten können. Dies gilt nicht für die KMW Deutschland, die aufgrund ihrer exportgetriebenen Wirtschaft stabilitätsorientiert ist und daher daran interessiert ist, kurzfristige Finanzströme einzuschränken. Dies spiegelte sich in der Stellungnahme von Bundeskanzlerin Angela Merkel zur Regulierung des Finanzsektors wider:

compared to industry, where people have a deep understanding of the products they deal with, financial markets are a lot more opaque. That has to change so that a country like Germany, which still produces a lot of industrial good, does not have to carry the economic risks (Barber et al. 2008).

Deutschland orientiert sich daher an der Verfügbarkeit langfristiger Finanzmittel, regulierten Arbeitsmärkten und einer umfassenden Koordinierung zwischen den Unternehmen. Infolgedessen entsprach die FTS-Förderung durch Deutschland dem anhaltenden regulatorischen Zögern der KMWs gegenüber der Marktliberalisierung, welches nach der Finanzkrise bestätigt wurde und an Dynamik gewann. Bundeskanzlerin Angela Merkel kritisierte das angloamerikanische Modell als verantwortlich für die Finanzkrise und argumentierte:

we need to think about the relationship between capital and risk [and] in terms of the rules, the transparency guidelines and the entire standardisation of financial markets, we still have a strongly Anglo-Saxon-dominated system (Barber et al. 2008).

Das britische System der Finanzregulierung folgte einem „light-touch“ Regulierungsansatz. Das übergeordnete Ziel des Regulierungsansatzes einer britischen Regierung gegenüber den Finanzmärkten bestand darin, die Wettbewerbsfähigkeit Londons zu verbessern, und bevorzugte daher ein ,,risk-based approach“. Die britische Regierung hat dafür gesorgt, dass London auch nach der Finanzkrise ein attraktiver Finanzstandort bleibt: „,no inspection without justification, no form filling without justification and no information requirements without justification, not just a light touch but a limited touch“ (Furness 2012). Während die Regierung 2011 erklärte, dass es ,at this challenging time, it is vital that we lay the foundations for long term economic growth [which] means harnessing the strength of our financial services sector“ (Hoban 2011), argumentierte David Cameron 2013, ,this government has done everything business has asked for (...) we should stand up for those strong businesses that we have; that is absolutely key to Britain's economic future“ (Cameron 2013). Dies entspricht daher der Tatsache, dass in Großbritannien 
die Interpretation der Finanzkrise lautete, dass die Krise „was principally a failure of the collective imagination of many bright people“ (Besley und Hennessy 2009, S. 3) und kein Versagen des Systems. Die britische Regierung bewertete daher die Stärkung der Finanzregulierung angesichts der Ausstattung ihrer Wirtschaft mit einem der wichtigsten globalen Finanzzentren. Dies bestätigt die Hauptannahmen über LMWs, die durch freie Marktströme und eine geringe Besteuerung von Markttransaktionen gekennzeichnet sind.

\section{Schlussfolgerung}

Diese Studie untersuchte den Vorschlag, die Finanzregulierung im Zusammenhang mit der Debatte über Finanztransaktionssteuern (FTS) oder der sogenannten RobinHood-Steuer zu stärken, und die damit einhergehenden divergierende politischen Reaktionen der deutschen und britischen Regierungen. Angesichts der Tatsache, dass die europäische Finanzliteratur nach der Krise einen Widerspruch zwischen einer Zunahme und einer Abnahme der demokratischen Politikgestaltung liest und über die Literatur reflektiert, in der finanzielle Interessen gegen die öffentliche Meinung und die Bedenken von NGOs gestellt werden, untersuchte diese Studie die beiden divergierenden Regierungspositionen, im angeführten Zeitraum unter der Annahme des erweiterten Kooperationsmechanismus. Diese Untersuchung zur Präferenzbildung zeigt, dass die Regierungen nicht in einem politischen innerstaatlichen Vakuum handelten, sondern offen für ein breites gesellschaftliches Publikum, Interessengruppenverbände, Gewerkschaften, Wähler*innen, NGOs waren, das unmittelbar von der Krise betroffen war, was zu einer demokratischen Entscheidungsfindung führte. Indem besonderes Augenmerk auf die Auswirkungen der Pluralität der Akteure gelegt wurde und die Bedeutung der Präferenzbildungsprozesse der Länder hervorgehoben wurde, bestand das Hauptziel der Studie darin, zu untersuchen, wer die Reaktionen dieser Regierungen während der FTS-Debatte bestimmt hat und warum finanzielle und nicht-finanzielle gesellschaftliche Akteure im Prozess der demokratischen Entscheidungsfindung entweder beachtet oder abgelehnt wurden.

Der Societal Approach und seine erklärenden Variablen, materielle Interessen, wertgestützte Ideen und nationale Institutionen, wurden angewendet, um zu berücksichtigen, wann diese von Bedeutung waren, wie sie interagierten und welche maßgeblich für die Gestaltung der FTS-Positionen dieser Regierungen waren. Hier werden die Kernhypothesen jeweils erläutert, gefolgt von den jeweiligen empirischen Ergebnissen. Es ist wichtig daran zu erinnern, dass das Ziel nicht darin bestand, zu veranschaulichen, ob eine erklärende Variable anstelle einer anderen wichtig ist, sondern sich auf die Bedingungen zu konzentrieren, unter denen jede einzelne Variable für die Gestaltung der Regierungspositionen entscheidender wird.

Die erste formulierte Hypothese konzentrierte sich auf die Interessengruppen und Gewerkschaften aus den Bereichen Finanzen und Industrie bei der Gestaltung der FTS-Positionen der britischen und deutschen Regierung. Die Stärkung der EU-Finanzregulierung würde sich direkt auf bestimmte Wirtschaftssektoren auswirken und zu Kosten-Nutzen-Berechnungen führen, die diese Akteure dazu veranlassten, sich für Lobbying einzusetzen, und somit die Präferenzbildung dominierten. Die vergleichen- 
de empirische Analyse der deutschen und britischen materiellen Interessen zeigt, dass diese Akteurstypen, sowie Gewerkschaften hinsichtlich ihrer FTS-Präferenzen gespalten waren. Aufgrund der Rolle, die sie in der Volkswirtschaft spielen und in der sie zu Beschäftigung, Export und Bruttowertschöpfung (BWS) beitragen, waren britische Finanz- und Wirtschaftsinteressenverbände mehr als ihr deutsches Pendant gegen eine Stärkung der finanziellen Integration. Aussagen von Vertreter*innen von Finanz- und Wirtschaftsverbänden sowie Veröffentlichungen belegen diese Reformopposition. Ihr Hauptanliegen, wenn keine globale Finanztransaktionssteuer eingesetzt wird: Eine Stärkung der Finanzintegration würde zur Migration von Finanzinstituten, zu verzerrenden Wettbewerbsbedingungen und zu einem stagnierenden Wirtschaftswachstum führen, was zu Arbeitslosigkeit und Kosten für Sparer*innen und Rentner*innen führen würde. Gewerkschaften, die aufgrund der Bedeutung der Arbeit für die Volkswirtschaft als materielle Interessen eingestuft wurden, unterstützten die FTS, da sie den Finanzsektor für die schlechte wirtschaftliche Lage verantwortlich machten, die Arbeitslosigkeit und immense Kosten für den Steuerzahler verursacht hatte. Die FTS würde bei der „Bestrafung“ des Finanzsektors die Einnahmen erhöhen und zu politischer Stabilität, wirtschaftlichem Wohlstand und sozialer Sicherheit beitragen.

Die zweite Hypothese lenkte die Aufmerksamkeit auf die Bedenken von Wähler*innen und NGOs, wenn die Stärkung der EU-Finanzregulierung grundlegendbedeutende und langfristige gesellschaftliche Erwartungen an die angemessene Rolle der Regierung bei der Steuerung der Wirtschaft beinhaltet. Der länderübergreifende Vergleich hat gezeigt, dass NGOs in Deutschland und Großbritannien eine einheitliche Position gegenüber der FTS hatten. Die Forderung nach Fragen der sozialen Gerechtigkeit wie Entwicklung und Umwelt sowie die Solidarität mit armen Ländern bei der Aufteilung der Hälfte der FTS-Einnahmen waren in beiden Präferenzbildungsprozessen von Bedeutung. Die deutsche und britische öffentliche Meinung zu marktfreundlichen und regulierungsfreundlichen Einstellungen war unterschiedlich: die deutschen Befragten unterstützten eher die Rolle der Regierung bei der Steuerung der Wirtschaft, während das Vertrauen der britischen Befragten in die Marktkräfte eine negative Korrelation mit der Stärkung der Finanzregulierung aufwies. Dies entspricht gleichermaßen dem hohen Prozentsatz der deutschen Befragten, die die Einführung von Finanztransaktionssteuer befürworten, und entspricht auch der weitgehend geteilten öffentlichen Meinung in Großbritannien zu diesem Thema.

Während die FTS einerseits regulatorische Auswirkungen auf Finanztransaktionen haben sollte, würde sie andererseits erhebliche Einnahmen für Entwicklung und Umwelt generieren. Ihr doppelter Zweck erklärt nicht nur issue salience und die Pluralität der Akteure im Zusammenhang mit den FTS-Debatten in Deutschland und Großbritannien, sondern erklärt auch das Vorhandensein sowohl von materiellem Interesse als auch von wertgestützten Ideen in den Prozessen zur Präferenzbildung.

Bisher hat diese Studie die Bedeutung beider erklärenden Variablen hervorgehoben. Dabei würde deutlich, dass nicht nur innerhalb dieser Variablen in beiden Ländern (materielle Interessen: Finanz- und Wirtschaftsbranche versus Gewerkschaften) oder nur in Großbritannien (wertgestützten Ideen: NGOs versus Wähler*innen) eine gewisse Konkurrenz herrschte. Ebenso können sich diese Variablen gegenseitig 
abschwächen oder verstärken. Die dritte Hypothese konzentrierte sich auf die Interdependenz der Variablen: Die Stärkung der Finanzregulierung würde sich direkt auf die Kosten-Nutzen-Berechnungen sowie auf die grundlegenden langfristigen gesellschaftlichen Erwartungen an die angemessene Rolle der Regierung bei der Steuerung der Wirtschaft auswirken. Die vergleichende empirische Untersuchung hat gezeigt, dass die Forderungen der Gewerkschaften von Finanz- und Industrieverbänden mit diesen kollidierten, so dass eine gewisse Abschwächung des Einflusses der Variablen stattfand. Diese Abschwächung viel umso mehr auf in der britischen Fallstudie, da die Gewerkschaft in Deutschland sowohl von Wähler*innen als auch von NGOs bei der FTS unterstützt wurden. Daher fand eine Wechselwirkung zwischen den erklärenden Variablen statt. Zudem wurde im britischen Präferenzbildungsprozess der Widerstand der Finanz- und Industrieinteressenverbände höchstwahrscheinlich durch die geteilte Meinung der Öffentlichkeit verstärkt. Da es nicht gelungen war, eine solide positive Haltung gegenüber der FTS einzunehmen, verstärkten die Einstellungen der Wähler*Innen die materiellen Interessen gegen die FTS. In Deutschland konnten Gewerkschaften, Wähler*innen und NGOs daher die Interessen der Finanz- und Wirtschaftsbranche umgehen und damit die Position ihrer Regierung zur Unterstützung der vorgeschlagenen Reform nachhaltig gestalten, während diese Akteure in Großbritannien letztendlich nicht in der Lage waren, einer einheitlichen Finanzindustrie, welche sich überwiegend gegen die FTS aussprach, entgegenzuwirken.

Die letzte Hypothese befasste sich mit der Frage der Stärkung der Finanzregulierung und Fragen zu formalen Regelungen der sozioökonomischen Koordinierung. Die Positionen der Regierungen würden mit den nationalen Institutionen übereinstimmen, da die Auswirkungen materieller Interessen und wertgestützter Ideen auf die Präferenzbildung der Regierung verstärkt werden, wenn sie nationalen ,institutional settings“ entsprechen (Schirm 2016, S. 69). Die länderspezifische Art der Marktwirtschaft hat ein Verständnis dafür vermittelt, dass die FTS-Positionen beider Regierungen im Einklang mit den LMW- und KMW-Rahmenbedingungen stehen. Die positive Haltung Deutschlands gegenüber der FTS korrelierte mit wertgestützten Ideen (und nicht mit sektoralen Interessen) im FTS-Reformvorschlag. In Bezug auf den FTS-Reformvorschlag verstärkte dies die Wirkung regulierungsfreundlicher Ideen und damit die Position der Regierung zugunsten des Reformvorschlags. Umgekehrt korrelierte die gegnerische Position der britischen Regierung mit materiellen Interessen (und nicht mit Gewerkschaften). Das britische LMW, ,lighttouch"-Regulierungssystem verstärkte die Auswirkungen materieller Interessen und wertgestützter Ideen, die der Einführung der FTS entgegenstanden (oder zumindest nicht dafür waren), und führte folglich dazu, dass die Regierung die FTS ablehnte.

Unter besonderer Berücksichtigung der Auswirkungen der issue salience und der Akteurspluralität fand die FTS-Debatte unter Beteiligung verschiedener finanzieller und nicht-finanzieller gesellschaftlicher Akteure statt. Unter Anwendung des Societal Approach und seiner drei Erklärungsvariablen wurde berücksichtigt, wann diese relevant waren, wie sie interagierten und welche bei der Gestaltung der deutschen und britischen Regierungspositionen prägend waren. Diese vergleichende theoriegeleitete empirische Analyse hat gezeigt, dass Gewerkschaften, Wähler*innen und NGOs in Deutschland die Interessen der Finanzindustrie bei der Gestaltung der Re- 
gierungsposition zur Unterstützung der vorgeschlagenen Reform umgehen konnten, während es diesen Akteuren nicht gelungen ist, einer einheitlichen Finanzindustrie in Großbritannien entgegenzutreten, die die FTS stark ablehnte. Ausgehend von der aktuellen Literatur zur europäischen Finanzregulierung nach der Krise, die sich mit einem gewissen Dissens zwischen einer Zunahme und einer Abnahme der demokratischen Politikgestaltung in Krisenzeiten befasst, hat diese Studie zur Identifizierung einer verstärkten demokratischen Politikgestaltung beigetragen und veranschaulicht, dass Regierungen auch in Krisenzeiten gegenüber einer Vielzahl gesellschaftlicher Akteure responsiv sind.

Danksagung Für hilfreiche Anmerkungen und Kommentare dankt die Autorin den anonymen Gutachterinnen und Gutachtern und den Herausgebern des Special Issue. Dank gebührt ferner Sandra Breslauer und Nancy Westermeyer.

Funding Open Access funding enabled and organized by Projekt DEAL.

Open Access Dieser Artikel wird unter der Creative Commons Namensnennung 4.0 International Lizenz veröffentlicht, welche die Nutzung, Vervielfältigung, Bearbeitung, Verbreitung und Wiedergabe in jeglichem Medium und Format erlaubt, sofern Sie den/die ursprünglichen Autor(en) und die Quelle ordnungsgemäß nennen, einen Link zur Creative Commons Lizenz beifügen und angeben, ob Änderungen vorgenommen wurden.

Die in diesem Artikel enthaltenen Bilder und sonstiges Drittmaterial unterliegen ebenfalls der genannten Creative Commons Lizenz, sofern sich aus der Abbildungslegende nichts anderes ergibt. Sofern das betreffende Material nicht unter der genannten Creative Commons Lizenz steht und die betreffende Handlung nicht nach gesetzlichen Vorschriften erlaubt ist, ist für die oben aufgeführten Weiterverwendungen des Materials die Einwilligung des jeweiligen Rechteinhabers einzuholen.

Weitere Details zur Lizenz entnehmen Sie bitte der Lizenzinformation auf http://creativecommons.org/ licenses/by/4.0/deed.de.

\section{Literatur}

Alt, Jörg. 2011. Stellungnahme des Hauptpetenten aus Anlass der Öffentlichen Anhörung der Petition „Einführung einer Finanztransaktionssteuer“ im Deutschen Bundestag. https://www.steuer-gegenarmut.org/fileadmin/Dateien/Kampagnen-Seite/Wer_wir_sind/Stellungnahmen/110207_Statement. pdf (Erstellt: 7. Febr. 2011). Zugegriffen: 11. Apr. 2020.

Bankenverband. 2013. Aktuelles Stichwort - Finanztransaktionssteuer - Wer zahlt? https://bankenverband. de/media/publikationen/aktuelles-stich_Mk56lu2.pdf (Erstellt: 22. Febr. 2013). Zugegriffen: 20. Sept. 2019.

Barber, Lionel, Bertrand Benoit, und Hugh Williamson. 2008. Regulation model has failed, says Merkel. Financial Times. https://www.ft.com/content/1f436388-371e-11dd-bc1c-0000779fd2ac (Erstellt: 11. Juni 2008). Zugegriffen: 5. März 2020.

Barker, Alex. 2013. EU blessing at core nation's transaction tax. Financial Times. https://www.ft.com/ content/66d35132-64ae-11e2-ac53-00144feab49a (Erstellt: 22. Jan. 2013). Zugegriffen: 2. Apr. 2020.

Bauer, Michael W., und Stefan Becker. 2014. The unexpected winner of the crisis: the European commission's strengthened role in economic governance. Journal of European Integration 36(3):213-229.

BBA. 2011. BBA Responds to the European Commission's Consultations on FinancialSector Taxation. www.bba.org.uk/policy/financial-and-risk-policy/taxation/fs-taxation/bbaresponse-to-theeuropean-commissionis-consultation-on-financial-sector-taxation-2/. Zugegriffen: 17. Okt. 2019.

Besley, Tim, und Peter P. Henessey. 2009. Letter to the Queen, London 22. Juli. https://wwwf.imperial.ac. uk/ bin06/M3A22/queen-lse.pdf. Zugegriffen: 3. Apr. 2020. 
Bickerton, Christopher J., Dermot Hodson, und Uwe Puetter. 2015. The New Intergovernmentalism: European Integration in the Post-Maastricht Era. Journal of Common Market Studies 53(4):703-722. https://doi.org/10.1111/jcms.12212.

Bundesministerium der Finanzen. 2020. Tax policy priorities of Germany's Council Presidency. German Federal Ministry of Finance. https:/www.eu2020finance.de/en/news/steuerpolitische-schwerpunkteder-deutschen-eu-ratspraesidentschaft. Zugegriffen: 18. Okt. 2020.

Bundesregierung. 2012. Pakt für nachhaltiges Wachstum und Beschäftigung - Gemeinsames Papier der Bundesregierung und der Fraktionen CDU/CSU, FDP, SPD und Bündnis 90/Die Grünen im Deutschen Bundestag, Pressemitteilung 21.Juni 2012. https://archiv.bundesregierung.de/resource/ blob/656922/744304/3d78b5c23bd0af2375ea935fe743dd90/2012-06-21-wachstum-pakt-data.pdf. Zugegriffen: 14. Juni 2019.

Bundesregierung. 2014. Finanztransaktionssteuer: Elf Mitgliedsstaaten gehen voran. https://www. bundesregierung.de/breg-de/aktuelles/elf-mitgliedstaaten-gehen-voran-420754 (Erstellt: 7. Mai 2014). Zugegriffen: 14. Juni 2019.

Cameron, David. 2012. PM Davos Speech Delivered on 26 January 2012. World Economic Forum. Davos. https://www.gov.uk/government/speeches/pm-davosspeech. Zugegriffen: 30. Sept. 2019.

Cameron, David. 2013. PM Global Investment Conference Speech Delivered on 9 May 2013, London. https://www.gov.uk/government/speeches/transcript-of-pms-speech-at-the-global-investmentconference. Zugegriffen: 30. Sept. 2019.

CBI. 2012. CBI welcomes government decision on financial transaction tax. www.cbi.org.uk/mediacentre/press-releases/2012/10/cbi-welcomes-governmentdecision-on-financial-transaction-tax/. Zugegriffen: 22. Nov. 2019.

CEPR. 2013. Statements of support for a financial transaction tax (FTT) updated Juli 2013. https://www. robinhoodtax.org.uk/sites/default/files/ftt-support.pdf. Zugegriffen: 21. März 2020.

Chaffin, Joshua, Stanley Pignal, und Jeremy Grant. 2011. Business attacks transaction tax plan, in Financial Times. https://www.ft.com/content/f9d2188a-e9ec-11e0-a149-00144feab49a (Erstellt: 28. Sept. 2011). Zugegriffen: 13. Febr. 2020.

City AM (2014) Boris slams transactions tax as a threat to the City, 30 April 2014. https://www.cityam. com/boris-slams-transactions-tax-threat-city/. Zugegriffen 12 Mai 2019.

COBCOE. 2011. To bin or not to bin. www.cobcoe.eu/wp-content/uploads/2011/10/PP1011.pdf. Zugegriffen: 11. Mai 2019.

Cordoro, Guillermo, und Pablo Simón. 2016. Economic crisis and support for democracy in Europe. West European Politics 39(2):305-325. https://doi.org/10.1080/01402382.2015.1075767.

Csehi, Robert, und Uwe Puetter. 2021. Who determined what governments really wanted? Preference formation and the euro crisis. West European Politics 44(21): 463-484. https://doi.org/10.1080/ 01402382.2020 .1731666

Culpepper, Pepper D. 2012. Quiet Politics and Business Power. Corporate Control in Europe and Japan. Cambridge: CUP.

DAI. 2013. Die Finanztransaktionssteuer - Ein Politischer Irrweg? Oliver Wyman. https://www.dai. de/files/dai_usercontent/dokumente/studien/2013-07-18\%20Deutsches\%20Aktieninstitut\%20Oliver \%20Wyman\%20FTS-Studie.pdf. Zugegriffen: 3. Apr. 2019.

De Bruycker, Iskander, und Jan Beyers. 2019. Lobbying strategies and success: Inside and outside lobbying in European Union legislative politics. European Political Science Review 11(1):57-74.

Degner, Hanno, und Dirk Leuffen. 2019a. Franco-German cooperation and the rescuing of the Eurozone. European Union Politics 20(1):89-108. https://doi.org/10.1177/1465116518811076.

Degner, Hanno, und Dirk Leuffen. 2019b. Crisis and responsiveness: analysing German preference formation during the Eurozone crisis. Political Studies Review https://doi.org/10.1177/1478929919864902.

Deutscher Bundestag. 2011. Finanztransaktionssteuer unter Experten umstritten. https://www.bundestag. de/dokumente/textarchiv/2011/36701097_kw48_pa_finanzen-207024 (Erstellt: 30. Nov. 2011). Zugegriffen: 5. Apr. 2019.

DGB. 2011a. Europa neu justieren - Wachstum fördern, Beschäftigung sichern, Euro stabilisieren. https:// www.dgb.de/++co++2b7d6dfa-2fb0-11e0-5ba7-00188b4dc422 (Erstellt: 3. Febr. 2011). Zugegriffen: 11. Mai 2019.

DGB. 2011b. Finanztransaktionssteuer muss jetzt kommen. https://www.dgb.de/presse/++co++c70105429291-11e0-4695-00188b4dc422 (Erstellt: 9. Juni 2011). Zugegriffen: 11. Mai 2019.

DGB. 2013. A marschall plan for Europe. https:/www.dgb.de/++co++d92f2d46-5590-11e2-832700188b4dc422 (Erstellt: 3. Jan. 2013). Zugegriffen: 11. Mai 2019.

DIHK, BDI, ZDH, BDA, BdB, GDV, HDE, und BGA. 2011. Stellungnahme - Öffentliche Anhörung zur Finanztransaktionssteuer. https://www.biv-kaelte.de/fileadmin/user_upload/mitglieder/ 
Rechtsberatung/Verbaende_Finanztransaktionssteuer.pdf (Erstellt: 23. Nov. 2011). Zugegriffen: 9. Mai 2019.

Dür, Andreas, und Gemma Mateo. 2012. Who lobbies the European Union? National interest groups in a multilevel polity. Journal of European Public Policy 19(7):969-987.

Dür, Andreas, und Gemma Mateo. 2016. Insiders versus outsiders: interest group politics in multilevel Europe. Oxford: OUP.

De Wilde, Pieter, Anna Leupold, und Henning Schmidtke. 2016. Introduction: the differentiated politicisation of European governance. West European Politics 39(1):3-22. https://doi.org/10.1080/01402382. 2015.1081505.

Eising, Rainer. 2009. The political economy of state-business relations in Europe capitalism, interest mediation, and EU-policy-making. London: Routledge.

Elliot, Larry, und Julia Kollewe. 2011. German finance minister says too many Gastarbeiter were allowed in. The Guardian. https://www.theguardian.com/world/2011/mar/18/german-finance-minister-guestworkers-row (Erstellt: 18. März 2011). Zugegriffen: 11. Apr. 2020.

Epstein, Lee, und Jeffrey A. Segal. 2000. Measuring issue salience. American Journal of Political Science 44:66-83.

Eurobarometer. 2011. Table of Results - Standard Eurobarometer 75 European Perceptions on the State of the Economy. https://ec.europa.eu/commfrontoffice/publicopinion/archives/eb/eb74/eb74_anx_en. pdf. Zugegriffen: 14. Apr. 2020.

Eurobarometer. 2012. Eurobarometer 78, Public Opinion in the European Union. http://ec.europa.eu/ commfrontoffice/publicopinion/archives/eb/eb78/eb78_anx_en.pdf. Zugegriffen: 14. Apr. 2020.

Eurobarometer. 2013. Eurobarometer 80, Europeans, The European Union and the Crisis. https://ec.europa. eu/commfrontoffice/publicopinion/index.cfm/Survey/getSurveyDetail/instruments/STANDARD/ yearFrom/1974/yearTo/2014/surveyKy/1123. Zugegriffen: 14. Apr. 2020.

European Commission. 2011. Financial transaction tax: making the financial sector pay its fair share. Pressemitteilung. https://europa.eu/rapid/press-release_IP-11-1085_en.htm?locale=en (Erstellt: 28. Sept. 2011). Zugegriffen: 11. Mai 2019.

Ezrow, Lawrence, und Timothy Hellwig. 2014. Responding to voters or responding to markets? Political parties and public opinion in an era of globalization. International Studies Quarterly 58(4):816-827.

Fabbrini, Sergio, und Uwe Puetter. 2016. Integration without supranationalism: studying the lead roles of the European Council and the Council in post-Lisbon EU politics. Journal of European Integration 38(5):481-495.

Falloon, M. 2011. EU financial transaction tax would be "suicide" - Osborne, Reuters Business News. https://uk.reuters.com/article/uk-britain-osborne/eu-financial-transaction-tax-would-be-suicideosborne-idUKTRE7AD1E820111114 (Erstellt: 11.11.). Zugegriffen: 7. Mai 2019.

FAZ. 2012. Finanzminister-Treffen: Transaktionssteuer kommt nicht in allen EU-Staaten. https://www.faz. net/aktuell/wirtschaft/finanzminister-treffen-transaktionssteuer-kommt-nicht-in-allen-eu-staaten11795448.html (Erstellt: 22. Juni 2012). Zugegriffen: 11. Mai 2019.

Fioretos, Orfeo. 2011. Historical Institutionalism in International Relations. International Organization 65(2):367-399.

Furness, Hannah. 2012. Regulating the banks: what politicians used to say about the City. The Telegraph. https://www.telegraph.co.uk/news/politics/9376534/Regulating-the-banks-what-politicians-usedto-say-about-the-City.html (Erstellt: 4. Juli 2012). Zugegriffen: 12. Apr. 2020.

Goldstein, Judith, und Robert O. Keohane. 1993. Ideas and foreign policy: beliefs, institutions and political change. Cornell: Cornell University Press.

Grossman, Gene M., und Elhanan Helpman. 1994. Protection for Sale. The American Economic Review 84(4):833-850. www.jstor.org/stable/2118033.

Hall, Peter A., und David Soskice. 2001. Varieties of capitalism: the institutional foundations of comparative advantage. Oxford: OUP.

Handelsblatt. 2012. „SZ“/EU: Finanztransaktionssteuer kann noch 2012 vereinbart werden. https://www. handelsblatt.com/dpa/economy-business-und-finance-sz-eu-finanztransaktionssteuer-kann-noch-

2012-vereinbart-werden/6742870.html?ticket=ST-7579869-26DwpFFugMSvJ1XnvTyz-ap6 (Erstellt: 12. Juni 2012). Zugegriffen: 5. Mai 2019.

Hardiman, Niamh, und Saliha Metinsoy. 2019. Power, ideas and national preferences: Ireland and the FTT. Journal of European Public Policy 26(11):1600-1619.

Hoban, Mark. 2011. Speech by the Financial Secretary to the Treasury, Mark Hoban MP, to APCIMS . https://www.gov.uk/government/speeches/speech-by-the-financial-secretary-to-the-treasury-markhoban-mp-to-apcims (Erstellt: 11. Okt. 2011). Zugegriffen: 6. Apr. 2020. 
Hoban, Mark. 2012. Letter to Lord Harrison, Chair of the Economic and Financial Affairs and International Trade Sub-Committee. https://www.parliament.uk/documents/lords-committees/eu-sub-coma/FinancialTransactionTax/FTTGovResponse.pdf (Erstellt: 24. Mai 2012). Zugegriffen: 5. Apr. 2020.

Hobolt, Sarah B., und Robert Klemmensen. 2008. Government responsiveness and political competition in comparative perspective. Comparative Political Studies 41(3):309-337. https://doi.org/10.1177/ 0010414006297169.

Hooghe, Liesbet, und Gary Marks. 2009. A postfunctionalist theory of European integration: from permissive consensus to constraining dissensus. British Journal of Political Science 39(1):1-23. https://doi. org/10.1017/S0007123408000409.

House of Lords. 2012. Financial Transaction Tax: Oral and Written Evidence. EU Economic and Financial Affairs and International Trade Sub-Committee. https://www.parliament.uk/documents/lordscommittees/eu-sub-com-a/FinancialTransactionTax/FTTWrittenandoralevidence.pdf. Zugegriffen: 5. Apr. 2020.

House of Lords. 2013. Financial transaction tax: alive and deadly. European Union committee, 7th report of session 2013-2014. London: STO. https://publications.parliament.uk/pa/ld201314/ldselect/ldeucom/ 86/86.pdf. Zugegriffen: 5. Apr. 2020.

Hutter, Swen, und Hanspeter Kriesi. 2019. Politicizing Europe in times of crisis. Journal of European Public Policy 26(7):996-1017.

ISDA. 2011. Joint association FTT letter to Chancellor of the Exchequer George Osborne. https://www. isda.org/a/2KDDE/joint-association-ftt-letter-24-10-11.pdf (Erstellt: 24. Okt. 2011). Zugegriffen: 5. Apr. 2020.

ISDA. 2012. Letter to Daily Telegraph. https://www.isda.org/a/svDDE/ftt-letter-to-daily-telegraph.pdf (Erstellt: 13. Febr. 2012). Zugegriffen: 5. Apr. 2020.

ITUC. 2012. Global Poll 2012-How banks can contribute to society: very strong popular support for financial transaction tax. https://www.ituc-csi.org/ituc-global-poll-2012-how-banks?lang=en (Erstellt: 18. Juni 2012). Zugegriffen: 6. Apr. 2020.

Kalaitzake, Manolis. 2017. Death by a thousand cuts? Financial political power and the case of the European financial transaction tax. New Political Economy 22(6):709-726. https://doi.org/10.1080/ 13563467.2017.1311850.

Kastner, Lisa. 2017. Civil society and financial regulation. Consumer finance protection and taxation after the financial crisis. London: Routledge.

Kastner, Lisa. 2018. Business lobbying under salience-financial industry mobilization against the European financial transaction tax. Journal of European Public Policy 25(11):1648-1666.

Keohane, Robert O., und Milner Helen V. (Hrsg.) (1996) Internationalization and Domestic Politics. Cambridge: Cambridge University Press.

Keller, Eileen. 2018. Noisy business politics: lobbying strategies and business influence after the financial crisis. Journal of European Public Policy 25(3):287-306.

Koalitionsvertrag. 2013. Deutschlands Zukunft gestalten. Koalitionsvertrag zwischen CDU, CSU und SPD, 18. Legislaturperiode. https://www.cdu.de/sites/default/files/media/dokumente/koalitionsvertrag. pdf. Zugegriffen: 3. Apr. 2019.

Kreuder-Sonnen, Christian. 2016. Beyond integration theory. The (anti-)constitutional dimension of European crisis governance. Journal of Common Market Studies 54(6):1350-1366. https://doi.org/10. $1111 /$ jcms. 12379.

Kreuder-Sonnen, Christian. 2018. Political secrecy in Europe: crisis management and crisis exploitation. West European Politics 41(4):958-980. https://doi.org/10.1080/01402382.2017.1404813.

Kriesi, Hanspeter. 2018. The implications of the euro crisis for democracy. Journal of European Public Policy 25(1):59-82. https://doi.org/10.1080/13501763.2017.1310277.

Milner, Helen V. 1997. Interests, institutions and information: domestic politics in international relations. Princeton: Princeton University Press.

Moravcsik, Andrew. 1993. Preferences and power in the European community: a liberal Intergovernmentalist approach. Journal of Common Market Studies 31(4):473-524. https://doi.org/10.1111/j.14685965.1993.tb00477.x.

Moravcsik, Andrew. 1997. Taking preferences seriously: a liberal theory of international politics. International Organization 51(4):513-553. https://doi.org/10.1162/002081897550447.

Niemann, Arne, und Demosthenes Ioannou. 2015. European economic integration in times of crisis: a case of neofunctionalism? Journal of European Public Policy 22(2):196-218.

OECD. 2020a. Employment by activity (indicator). https://data.oecd.org/emp/employment-by-activity. htm. Zugegriffen: 9. Apr. 2020. 
OECD. 2020b. Value added by activity (indicator). https://data.oecd.org/natincome/value-added-byactivity.htm. Zugegriffen: 9. Apr. 2020.

Oxfam. 2011. Robin Hood, Robin Hood, dum dum dum de dum: financial transaction tax update from Max Lawson. https://oxfamblogs.org/fp2p/robin-hood-robin-hood-dum-dum-dum-de-dum-thefinancial-transaction-tax-guest-post-from-max-lawson/ (Erstellt: 14. Apr. 2011). Zugegriffen: 8. Apr. 2020.

Parker, George. 2014. Osborne set for defeat over financial transaction tax. Financial Times. https://www.ft. com/content/e3a40298-cfaf-11e3-9b2b-00144feabdc0 (Erstellt: 29. Apr. 2014). Zugegriffen: 3. Apr. 2020.

Pew Research Center. 2012. European Unity on the Rocks. https://www.pewresearch.org/wp-content/ uploads/sites/2/2012/05/Pew-Global-Attitudes-Project-European-Crisis-Report-FINAL-FORPRINT-May-29-2012.pdf (Erstellt: 29.05.). Zugegriffen: 5. Apr. 2019.

Puetter, Uwe. 2012. Europe's deliberative intergovernmentalism: the role of the Council and European Council in EU economic governance. Journal of European Public Policy 19(2):161-178.

Quaglia, Lucia. 2017. European Union financial regulation, banking union, capital markets union and the UK. SPERI Paper No. 38. Sheffield: SPERI, University of Sheffield.

Robin Hood Tax. 2011a. There is an alternative-time for a Robin Hood tax. Policy brief. https://www. robinhoodtax.org.uk/sites/default/files/There_is_an_Alternative_0.pdf. Zugegriffen: 5. Apr. 2020.

Robin Hood Tax. 2011b. David Cameron promises to "safeguard" city interests in Europe. https://www. robinhoodtax.org.uk/david-cameron-promises-\%E2\%80\%98safeguard\%E2\%80\%99-city-interestseurope (Erstellt: 8. Dez. 2011). Zugegriffen: 10. Apr. 2020.

Rothacher, Jan-Ulrich. 2015. How domestic politics shaped the French government's position during the euro crisis. European Politics and Society 16(2):256-279.

Sanders, David, und Gabor Toka. 2013. Is anyone listening? mass and elite opinion cueing in the EU. Electoral Studies 32:13-25. https://doi.org/10.1016/j.electstud.2012.10.001.

Schiffers, Maximilian, und Annika Körner. 2019. NGOs in Prozessen der politischen Interessenvermittlung. Zeitschrift für Politikwissenschaft 29:525-541.

Schimmelfennig, Frank. 2015. Liberal Intergovernmentalism and the euro area crisis. Journal of European Public Policy 22(2):177-195.

Schirm Stefan, A. 2009. Ideas and interests in global financial governance: Comparing German and US preference formation. Cambridge Review of International Affairs, 22(3): 501-521. https://doi.org/10. 1080/09557570903103992

Schirm, Stefan A. 2013. Global politics are domestic politics: a societal approach to divergence in the G20. Review of International Studies 39(3):685-706. https://doi.org/10.1017/S0260210512000216.

Schirm, Stefan A. 2016. Domestic ideas, institutions or interests? Explaining governmental preferences towards global economic governance. International Political Science Review 37(1):66-80. https:// doi.org/10.1177/0192512114540190.

Schirm, Stefan A. 2018. Societal foundations of governmental preference formation in the Eurozone crisis. European Politics and Society 19(1):63-78. https://doi.org/10.1080/23745118.2017.1340397.

Schirm, Stefan A. 2020. Refining domestic politics theories of IPE: a societal approach to governmental preferences. Politics 40(4):396-412. https://doi.org/10.1177/0263395719896980.

Schirm Stefan, A. 2011. Varieties of strategies: societal influences on British and German responses to the global economic crisis. Journal of Contemporary European Studies 19(1):47-62. https://doi.org/10. 1080/14782804.2010.535716.

Schmidt, Vivien A. 2015. The Eurozone's crisis of democratic legitimacy. Can the EU rebuild public trust and support for European economic integration? European economy discussions paper no. 15 (European commission). https://ec.europa.eu/info/sites/info/files/dp015_en.pdf. Zugegriffen: 5. Juli 2019.

Schulmeister, Stephan, Margit Schratzenstaller, und Oliver Picek. 2008. A general financial transaction tax. Motives, revenues, feasibility and effects. Österreichisches Institut für Wirtschaftsforschung. Vienna. https://www.wifo.ac.at/jart/prj3/wifo/resources/person_dokument/person_dokument.jart? publikationsid=31819\&mime_type=application/pdf. Zugegriffen: 14. Apr. 2020.

Smeets, Sandrino, und Derek Beach. 2019. Political and instrumental leadership in major EU reforms. The role and influence of the EU institutions in setting-up the Fiscal Compact. Journal of European Public Policy https://doi.org/10.1080/13501763.2019.1572211.

Stewart, Heather. 2011. World economists urge G20 ministers to accept Robin Hood tax. The Guardian. https://www.theguardian.com/business/2011/apr/13/world-economists-robin-hood-tax?INTCMP =SRCH (Erstellt: 13. Apr. 2011). Zugegriffen: 9. Apr. 2020. 
Van Loon, Aukje. 2018. Domestic politics and national differences in restructuring EU financial supervision. European Politics and Society 19(3):247-263. https://doi.org/10.1080/23745118.2017.1419598.

Van Loon, Aukje. 2020. Societal dynamics in European economic governance: a comparative analysis of variation in British and German governmental stances. In The future of global economic governance: challenges and prospects in the age of uncertainty, Hrsg. Marek Rewizorski, Karina Jędrzejowska, und Anna Wróbel, 119-139. Cham: Springer.

Van Loon, Aukje. 2021. Political contestation and domestic politics in EU financial regulation. In Democratic citizenship in flux: conceptions of citizenship in the light of political and social fragmentation, Hrsg. Markus Bayer, Oliver Schwarz, und Toralf Stark. Bielefeld: Transcript.

Verdun, Amy. 2017. Political leadership of the European central bank. Journal of European Integration 39(2):207-222.

Wahl, Peter. 2014. The European civil society campaign on the financial transaction tax. Global labour university working papers no. 20. http://www.global-labour-university.org/fileadmin/GLU_Working_ Papers/GLU_WP_No.20.pdf. Zugegriffen: 20. Febr. 2020.

Wearden, Graeme. 2011. European markets hit by eurozone Robin Hood tax plans. The Telegraph. https://www.theguardian.com/business/2011/aug/17/european-markets-hit-robin-hood-tax (Erstellt: 18. Aug. 2011). Zugegriffen: 4. März 2020.

Weiler, Joseph H.H. 2012. In the face of crisis: input legitimacy, output legitimacy and the political messianism of European integration. West European Politics 34(7):825-841. https://doi.org/10.1080/ 07036337.2012.726017.

Winnet, Robert, Victoria Ward, und Richard Alleyne. 2011. Archbishop of canterbury Rowan Williams calls for new tax on bankers. https://www.telegraph.co.uk/news/religion/8863794/Archbishop-ofCanterbury-Rowan-Williams-calls-for-new-tax-on-bankers.html (Erstellt: 1. Nov. 2011). Zugegriffen: 12. Jan. 2020.

World Bank. 2020. Insurance and financial services (\% of service exports, BoP). https://databank. worldbank.org/reports.aspx ?source=2\&series=BX.GSR.INSF.ZS\&country=. Zugegriffen: 9. Apr. 2020.

World Value Survey. 2006. World value survey wave 2005-2009, Online analysis. http://www.worldvalues survey.org/WVSOnline.jsp. Zugegriffen: 3. Jan. 2020.

Zimmermann, Hubert. 2010. Varieties of global financial governance? British and German approaches to financial market regulation. In Global finance in crisis: the politics of international regulatory change, Hrsg. Eric Helleiner, Stefano Pagliari, und Hubert Zimmermann, 121-136. New York: Routledge. 